\begin{tabular}{|c|c|c|}
\hline \multicolumn{3}{|c|}{$\begin{array}{l}\text { Anno 12, numero } 2 \quad \text { Maggio - Agosto } 2017 \\
\text { Poste Italiane - Spedizione in abbonamento postale - 70\% aut. DCB / Genova nr. } 569 \text { anno } 2005 \\
\text { TESTATA INDIPENDENTE CHE NON PERCEPISCE CONTRIBUTI PUBBLICI (legge 250/1990) }\end{array}$} \\
\hline \multicolumn{3}{|r|}{ Si ringrazia } \\
\hline \multicolumn{2}{|r|}{ IN QUESTO NUMERO } & \multirow{4}{*}{$\begin{array}{l}\text { Risk Management Magazine } \\
\text { Anno 12, n } 2 \text { Maggio - Agosto } 2017 \\
\text { Direttore Responsabile: } \\
\text { Maurizio Vallino } \\
\text { Condirettore } \\
\text { Corrado Meglio } \\
\text { Consiglio scientifico } \\
\text { Giampaolo Gabbi (Direttore del Consiglio Scientifico), } \\
\text { Paola Bongini, Anna Bottasso, Ruggero Bertelli, } \\
\text { Ottavio Caligaris, Simona Cosma, Paola Ferretti, } \\
\text { Andrea Giacomelli, Adele Grassi, Pier Giuseppe } \\
\text { Giribone, Duccio Martelli, Pasqualina Porretta, Anna } \\
\text { Grazia Quaranta, Enzo Scannella, Cristiana Schena, } \\
\text { Giuseppe Torluccio } \\
\text { Comitato di redazione } \\
\text { Emanuele Diquattro, Fausto Galmarini, Rossano } \\
\text { Giuppa, Aldo Letizia, Paolo Palliola, Enzo Rocca, } \\
\text { Fabio Salis }\end{array}$} \\
\hline & & \\
\hline 3 & $\begin{array}{l}\text { Commento al Consultative Document del } \\
\text { Comitato di Basilea "Simplified alternative } \\
\text { to the standardised approach to market risk } \\
\text { capital requirements" }\end{array}$ & \\
\hline 5 & $\begin{array}{l}\text { IFRS 9, Stress Testing, ICAAP: a } \\
\text { comprehensive framework for PD } \\
\text { calculation } \\
\text { di Carlo Toffano, Francesco Nisi and Lorenzo } \\
\text { Maurri }\end{array}$ & \\
\hline 12 & $\begin{array}{l}\text { Lo Standardised Measurement Approach } \\
\text { (SMA) per i rischi operativi } \\
\text { di Giacomo Petrini e Camillo Giliberto }\end{array}$ & $\begin{array}{l}\text { Vignettista: Silvano Gaggero } \\
\text { Proprietà, Redazione e Segreteria: } \\
\text { Associazione Italiana Financial Industry Risk } \\
\text { Managers (AIFIRM), Via Sile 18, } 20139 \text { Milano } \\
\text { Registrazione del Tribunale di Milano } \text { n }^{\circ} 629 \text { del }\end{array}$ \\
\hline 26 & \multirow[t]{2}{*}{$\begin{array}{l}\text { I paradigmi di apprendimento non } \\
\text { supervisionato per reti neurali in campo } \\
\text { finanziario: progettazione di self- } \\
\text { organizing maps per il rintracciamento di } \\
\text { anomalie di mercato } \\
\text { di Pier Giuseppe Giribone e Alessia Cafferata }\end{array}$} & $\begin{array}{l}\text { 10/9/2004 } \\
\text { ISSN 2283-7329 } \\
\text { E-mail: segreteria@aifirm.it; } \\
\text { Tel. } 3896946315 \\
\text { Stampa: Algraphy S.n.c. - Passo Ponte Carrega 62-62r } \\
\text { 16141 Genova }\end{array}$ \\
\hline & & $\begin{array}{l}\text { Le opinioni espresse negli articoli impegnano } \\
\text { unicamente la responsabilità dei rispettivi autori } \\
\text { SPEDIZIONE IN ABBONAMENTO POSTALE AI } \\
\text { SOCI AIFIRM RESIDENTI IN ITALIA, IN } \\
\text { REGOLA CON L'ISCRIZIONE }\end{array}$ \\
\hline
\end{tabular}




\section{Processo di referaggio degli articoli proposti per la pubblicazione}

Gli articoli che sono proposti alla rivista per la pubblicazione sono sottoposti in forma anonima a due successivi livelli di referaggio.

Il primo livello di referaggio (di ammissibilità) viene effettuato sull'articolo dai membri del Consiglio Scientifico che ne valutano la conguità ai temi trattati dalla rivista.

Il secondo livello di referaggio (di pubblicabilità) viene effettuato sull'articolo da due referee scelti all'interno del Consiglio Scientifico o all'esterno tra accademici, ricercatori, esperti della materia, che ne valutano il contenuto e forma.

\section{Regolamento redazionale}

"Risk Management Magazine" è il periodico di AIFIRM (Associazione Italiana Financial Istitutions Risk Managers) ed interamente dedicato ai temi del risk management.

La sua struttura organizzativa prevede, oltre al direttore responsabile, un condirettore e un Consiglio Scientifico formato da accademici; quest'ultimo è garante delle qualità e correttezza degli articoli pubblicati.

La rivista favorisce la diffusione di tutti i contenuti afferenti i temi del risk management, dagli aspetti normativi a quelli organizzativi e alle technicalities e vaglierà con interesse, per mezzo del Comitato Scientifico, i contributi che le perverranno.

Gli articoli proposti dovranno pervenire in formato Microsoft Word carattere Times New Roman 10 ed avere un numero di battute compreso tra 6.000 e 20.000; è gradita la presenza di tabelle e grafici così come la bibliografia.

Si richiede la predisposizione di un Absract in lingua inglese.

Le opinioni espresse negli articoli impegnano unicamente la responsabilità dei rispettivi autori 


\section{Basel Committee on Banking Supervision \\ Consultative Document Simplified alternative to the standardised approach to market risk capital requirements - June 2017}

La Commissione Aifirm coordinata dal Professor Umberto Cherubini e dal dottor Marco Bianchetti ha elaborato il commento di seguito esposto al Consultative Document del Comitato di Basilea "Simplified alternative to the standardised approach to market risk capital requirements".

Il documento è stato approvato il 18/9/2017 dal Comitato Tecnico Scientifico di Aifirm e il 25/9/2017 dal Consiglio di Aifirm.

\section{Introductory Remarks}

We welcome the opportunity to comment on the proposed simplified alternative to the standardized approach to market risk capital requirements (R-SbM).

In our view, the consultative document issued by the BCBS represents an important step to address a concrete concern related to the new standardized approach to market risk capital requirements (SbM), namely the fact that it might be too complex for smaller and less sophisticated banks. Having said that, the attempt to design a simplified version of the new standardized approach entails some significant challenges, which would require further analysis in order to assess:

- the potential consequences of the proposal on the aim to maintain a level playing field between large and small banks and between banks active in different jurisdictions

- the overall coherence of the R-SbM, in terms of proposed parameters, with the SbM

- the extent to which the simplified proposal actually entails lower implementation efforts.

In this view, we support the alternative option mentioned by the Committee of retaining a recalibrated version of the Basel II Standardised approach rather than adopting R-SbM.

\section{Specific comments}

The structure of eligibility criteria for the R-SbM includes several conditions that should be effective in isolating only banks where trading is neither sophisticated nor carried-out on a large scale. However, quantitative parameters seem to be very low and might limit significantly the overall ability of the proposal to reach its goals.

In particular, we point out that, for the $5 \%$ threshold that refers to risk-weighted assets, it might happen that a bank ceases to fulfill the requirement as an effect of risk weighted assets for credit and operational risks dropping, without any change in the absolute level of risk-weighted assets for market risk. Since the threshold level seems to be very low, it seems likely that many banks will be rather close to its level, increasing the probability of this type of event. This concern could be addressed either by setting the corresponding level higher than $5 \%$ of risk-weighted assets, or using a different threshold, e.g. based on common equity tier 1 capital without considering the other RWAs.

In addition, for banks breaching the threshold we suggest:

- $\quad$ to consider a monitoring period for triggering the breach (e.g. 3 or 4 quarterly calculation of RWAs and Own Funds) and then being mandated to shift from R-SbM to SbM;

- $\quad$ to permit an implementation period (e.g. 12 or 18 months) during which the bank will work on implementation of SbM but still applying R-SbM, as otherwise banks should be ready anyway (i.e. despite of the possibility to apply R$\mathrm{SbM}$ ) with SbM in advance and therefore the benefit would be largely reduced.

As a further remark, we observe that the consultative document doesn't seem to specify if the eligibility criteria for the R-SbM should be applied at the consolidated level or subsidiary level. Our understanding is that they are meant to be applied at the consolidated level, however we encourage the BCBS to consider the alternative of an entity level approach, which might be more appropriate for groups that comprise several small banks carrying out trading activities on a small scale. If an entity level approach is allowed, RWAs calculated by subsidiaries under the R-SbM could be, in principle, aggregated through a "building block" approach with RWAs calculated by other legal entities in the same group using other methods.

The consistency of application of the R-SbM across jurisdictions is another point of concern, given that the use of R-SbM is subject to supervisory approval and oversight. Different approaches by national supervisors might entail different costs for small banks in different jurisdiction and have negative consequences on the creation of a level playing field.

Coming to the actual differences between the proposed R-SbM and the SbM, namely:

- removal of vega and curvature risk components

- correlation scenarios simplification

- basis risk calculation simplification

- lower granularity for risk factors, coupled with a different set of parameters

we notice that one of the main challenges and costs for banks implementing the SbM is the calculation of sensitivities, even first order ones. While the removal of vega and curvature risk components will certainly remove some of the complexity, the fact that the R-SbM is still sensitivity-based will require significant changes for small banks in terms of systems, processes 
and human resources. In this respect, we think that further cost-benefit analysis might be useful involving the BCBS and, possibly, a panel of small banks.

Lower risk factor granularity seems to come at the price of a significant change in risk weights and correlations assumptions. While a certain amount of additional conservatism can be justified, we see the risk that the coherence of the R-SbM with the SbM gets lost. We think that it might be worthwhile for the BCBS to carry out a quantitative assessment of the consistency between the R-SbM and the SbM for sample portfolios and publish the results, rather than leaving this type of simulation to the industry only. Since the R-SbM will not be an option for large banks, it is unlikely that they can devote resources to analyze in detail how it works compared to the SbM. Once vega and curvature risk are set aside, it might be argued that the additional cost for banks associated with retaining the SbM risk factor granularity might be very low. Therefore, we suggest to consider an alternative approach under which the R-SbM maintains the same risk factors as the SbM, but also the same quantitative parameters, or very similar ones.

Commissione Aifirm Market Risk 


\section{IFRS 9, Stress Testing, ICAAP: a comprehensive framework for PD calculation}

Carlo Toffano, Francesco Nisi and Lorenzo Maurri (Prometeia)

\section{ABSTRACT}

In order to fulfil all the different requirements coming from competent authorities, Financial Institutions are asked to compute probabilities of default (PD) in several ways. For instance, a stressed Point in Time PD is required for stress-testing purposes to calculate impairment losses, a Through the Cycle PD is needed for RWA calculation while forward looking lifetime PDs are needed for the new IFRS 9 accounting standard compliance. This plurality gives rise to the need for synergisms among the different PD calculations methods in order to minimize the development and maintenance costs of PD models. Furthermore, synergies are required to both keep coherence among PD calculation methodologies, and address those requirements pressing for high integration between different regulatory frameworks (as can be seen from latest EBA guidelines on the 2018 EU-wide Stress Test that will include IFRS 9 methodology).

To this end, we propose a comprehensive framework addressing some of the main problems arising in developing PDs for regulatory purposes, such as how to: derive conditional Point in Time PDs, include macroeconomic scenario, develop forward looking transition matrix, calculate stressed Point in Time PDs and compute forward looking lifetime PDs for stress testing and IFRS 9 purposes. We found that variations in both the long-term convergence transition matrix and convergence parameter provide the required flexibility in the cumulative PD curves to guarantee high representative accuracy. We believe that such a framework will provide practitioners with a powerful tool useful to minimize the models' maintenance costs, facilitate the supervisory review process, and guarantee the coherence with currently used PD models, IT architecture and managerial processes. From a practical point of view, we propose 10 operational steps in the same methodological framework useful to address many of the problems faced by banks when accomplishing with IFRS 9, Stress Testing and ICAAP requirements.

Keywords: Credit Risk, IFRS 9, Stress Testing, ICAAP, PD, Merton, Markov, migration matrices.

\section{Introduction}

Starting from Basel II introduction in the first decade of 2000, steady-state, stable parameters have been required to banks for the calculation of risk-weighted assets (European Parliament, 2013). The objective of the authorities was to produce stable capital requirements, so avoiding procyclicality of regulatory capital requirements (see for example Repullo et al., 2009).

Those parameters are usually known as "Through-the-Cycle" (TTC) average PDs and represent an "average" or prudential PD over the entire business cycle. Basel risk parameters are also characterized by margins of conservatorism, so they can be interpreted as the bank's best estimate plus a conservative add-on (Van Gestel, 2014).

Later, regulatory or accounting evolution moved from prudential long-run estimates towards pure best estimates approaches trying to capture the actual underlying risk of financial assets at a specific point in time. This extended approach is reflected both in provisioning rules (IFRS 9) and in stress testing exercises (regulatory stress test, SREP stress test).

As a result of this evolution, banks are now required to provide probabilities of default (PD) in several forms, from stressed/unstressed PD to change in the rating philosophy itself (PIT vs TTC) and 12 months or lifetime PD. In particular, the new IFRS 9 accounting standard involves PIT parameters including forward-looking perspective (BCBS, 2015).

It is clear the strong similarity of concepts required by IFRS 9, stress testing and ICAAP, which rises the need for synergism between efforts made for PD calculations. Moreover, latest EBA methodological guidelines on EU-wide Stress Test (EBA, 2017) explicitly includes the IFRS 9 provisioning impact, so providing a regulatory incentive for an immediate integration between different frameworks.

This means that while financial institutions generally have TTC risk parameters developed for risk-weighted assets purposes, they are asked to provide PIT risk parameters for different regulatory requirements, too.

The process is complex, and there are two points to be stressed: first, rating models developed for IRB purposes might be a good starting point provided they can be reconciled to IFRS 9 PDs, and second, there is a clear similarity between regulatory requirements for IFRS 9, Stress Test and ICAAP which ask for estimates characterized by high responsiveness to current and forward looking macroeconomic scenarios. 
In this context, the challenge faced by banks is to produce regulatory compliant as well as coherent PD estimates while minimizing the amount of resources devoted to their development and maintenance.

Our paper proposes a simple and comprehensive framework for PD calculation aimed at satisfying the need for synergism between efforts made for PD calculations according to different regulatory requirements. It would also enable banks to easily develop Stress Test framework coherently with latest EBA guidelines (EBA, 2017) for what concerns PDs estimation, and IFRS 9. Our proposed framework is based on the widely used Merton model, thus it is founded on sound methodology while remaining simple enough to be clearly communicated during the supervisory review process.

\section{Theoretical framework}

This section describes the details of our theoretical framework. It is divided into ten subsections, one for each of the main problems faced by Banks in accomplishing regulatory requirements for PD calculations.

Our methodology is based on the assumption that internal rating models are already in place within the Bank. Starting from such models banks have to:

1. obtain PiT PDs and long term PDs from already existing PDs;

2. link default dynamics to the macroeconomic cycle;

3. include scenario dependency into bank portfolio pit PDs;

4. calculate migration probabilities scenario dependent;

5. converge to long term equilibrium;

6. guarantee coherence between lifetime PDs and stressed PDs;

7. forecast scenario dependent PDs and long-term PDs;

8. forecast scenario dependent and long-term migration probabilities;

9. derive scenario dependent lifetime PDs;

10. assess the fitting of the estimated PD curves.

\section{step 1: obtain PiT PDs and long term PDs from already existing PDs}

The first problem to be addressed is the derivation of PIT PDs required by both new accounting standards and stress test exercises.

There are several methods that can be used to transform TTC PDs into PIT PDs, but beyond the methodology used, how can we formally represent them since they are conditioned to current economic cycle?

Retrieving the Merton model for conditional PD modeling (Merton 1974; Vasicek 2002), we can define PD as the probability that the creditworthiness index of the firm falls below a certain threshold.

$P D_{i(t)}=\operatorname{Pr}\left(y_{i(t)} \leq c_{i}\right)$

Where:

$i=\mathrm{i}$-th company

$t=$ time instant

$y_{i(t)}=\alpha_{i} z_{k(t)}+\sqrt{1-\alpha_{i}^{2}} \varepsilon_{i(t)}$

$z_{k(t)}=\sum_{j=1}^{I} \beta_{k j} X_{j(t)}+\eta_{k(t)}$

$k=$ portfolio cluster for systematic component

$c_{\mathrm{i}}=$ default threshold

All random variables are assumed to be distributed as standard normal $(N(0,1))$, except macroeconomic factors since they have given correlation matrix. So, the obligor-specific creditworthiness $y_{i(t)}$ is a function of:

- Systematic component $\left(z_{k(t)}\right)$ : representing the economic system effect;

- Idiosyncratic component $\left(\varepsilon_{i\left[t_{j}\right)}\right)$ : obligor-specific effect;

- Single firm correlation to systematic components $\left(\alpha_{\mathrm{i}}\right)$.

The systematic component $z_{k(t)}$ is in turn a function of:

- Macroeconomic component $\left(X_{j(\mathrm{t})}\right)$ : macroeconomic factors effect;

- Cluster-specific component $\left(\eta_{k(t)}\right)$ : idiosyncratic cluster-specific effect;

- Factor loadings $\left(\beta=\left[\beta_{k 1}, \beta_{k 2}, \ldots, \beta_{k j}\right]\right)$ and factor to factor correlation structure $(\Sigma)$. 
So, PD conditional to current cycle is:

$$
P D_{i(t)}=\operatorname{Pr}\left(y_{i(t)} \leq c_{i}\right)=\operatorname{Pr}\left(\varepsilon_{i(t)} \leq \frac{c_{i}-\alpha_{i} z_{k(t)}}{\sqrt{1-\alpha_{i}^{2}}}\right)
$$

To design the lifetime forward looking PD path for IFRS9 purposes, banks should provide long term PDs and lead a convergence of the path in the long term. Several approaches can be implemented (e.g. Rubtsov and Petrov 2016, UK FSA 2007). Due to the need of synthesis, we assume the availability of a PIT PD and a long run PD that will be used in our framework.

\section{step2: link default dynamics to the macroeconomic cycle}

Stress testing exercises and IFRS9 require the construction of a satellite model linking the default risk to macroeconomic factors dynamics guaranteeing at the same time extreme sensitivity to the scenario provided by supervision authorities (see EBA, 2016).

Satellite models may be based on several formulations. Here we use a standard two steps ECM (Engle and Granger, 1987).

Short-term equation:

$\Delta z_{k(t)}=\sum_{j=1}^{J} \beta_{0, k j} \Delta X_{j(t)}+(\gamma-1) * v_{k(t-1)}+\eta_{k(t)}$

Equilibrium equation:

$z_{k(t)}=\sum_{j=1}^{I} \beta_{0, k j} X_{j(t)}+v_{k(t)}$

step3: include scenario dependency into bank portfolio pit PDs

Starting from the result obtained in step1, we can derive the default threshold $c_{i}$ as a function of PD and systematic factor.

$c_{\mathrm{i}}=\sqrt{1-\alpha_{\mathrm{i}}^{2}} \Phi^{-1}\left(P D_{\mathrm{i}(\mathrm{g})}\right)+\alpha_{\mathrm{i}} z_{k(\mathrm{i})}$

This fixed default threshold can be substituted into the Merton formula to iteratively derive future conditional PDs $\left(P D_{\mathrm{i}(t)}\right.$, $\left.P D_{\mathrm{i}(\mathrm{t}+1)}, \ldots\right)$ :

$$
P D_{\mathrm{i}(\mathrm{t})}=\Phi\left[\Phi^{-1}\left(P D_{\mathrm{i}(\mathrm{t}-1)}\right)-\frac{\alpha_{\mathrm{i}}}{\sqrt{1-\alpha_{\mathrm{i}}^{2}}}\left(\Delta z_{k(t)}\right)\right]
$$

\section{step4: calculate migration probabilities scenario dependent}

EBA Stress Testing and IFRS 9 framework request that portfolio risk dynamic should be led by transition matrices conditioned by the macroeconomic scenario.

The passages developed at PD level can be easily extended to the migration matrix case. Suppose to have an obligor with rating $r$, then the migration probabilities on a generic rating class $m$ are:

$q_{r_{2}, t}=\operatorname{Pr}\left(c_{r_{2} m} \leq y_{\mathrm{ier}, t} \leq c_{r_{2} m+1}\right)$

Where $q_{r_{3}, t}$ is the rating migration probability (for the ith obligor belonging to rating class $r$ at time $t$ ) to a rating class bounded by threshold $m$ and $m+1$.

Graphically, it represents the area under the PD density curve for an asset with initial rating class $r$. 


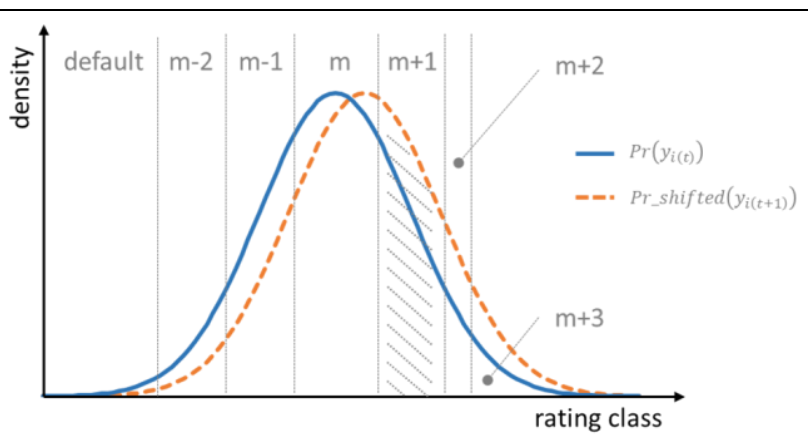

Figure 1 - Migration probabilities from initial rating class $r$

The migration probability from rating class $r$ is the probability that the creditworthiness factor lies between two thresholds. From the previous formula it can be shown that:

$q_{r_{m} m}=\Phi\left(\frac{c_{r_{i} m+1}-\alpha_{i} z_{k(t)}}{\sqrt{1-\alpha_{i}^{2}}}\right)-\Phi\left(\frac{c_{r_{m} m}-\alpha_{i} z_{k(t)}}{\sqrt{1-\alpha_{i}^{2}}}\right)=P_{r_{m} m+1, t}-P_{r_{m} m t}$

Both $c_{\gamma_{m} m}$ and $c_{Y m+1}$ can be obtained as in the PD case. By substituting these two thresholds, it is possible to derive migration probabilities at time $t$ as $q_{r_{2}, m_{t}}$ :

$q_{r_{i} m_{i} t}=\Phi\left[\Phi^{-1}\left(P_{r_{2} m+1, t-1}\right)-\frac{\alpha_{i}}{\sqrt{1-\alpha_{i}^{2}}}\left(\Delta z_{k(t)}\right)\right]-\Phi\left[\Phi^{-1}\left(P_{r_{2} m_{i} t-1}\right)-\frac{\alpha_{i}}{\sqrt{1-\alpha_{i}^{2}}}\left(\Delta z_{k(t)}\right)\right]$

What we obtained is a relation that links migration probabilities of the PIT transition matrix at time $t$ to the macroeconomic forecasts in $t$ provided by the satellite model seen in step2, and the rules to use them to forecast the migration probabilities.

\section{step5: converge to long term equilibrium}

The typical need in lifetime PD calculation for IFRS9 is to hypothesize long-term convergence of "future" PDs; in our framework this implies a convergence of the systematic factor $z$.

Under the hypothesis of ECM dynamics, we include long-run convergence of the systematic factor $z_{k(t+1)}$ as:

$\Delta z_{k(t+1)}=\sum_{j=1}^{J} \beta_{0, k j} \Delta X_{j(t+1)}+(\gamma-1)\left(z_{k(t+1-1)}-\bar{z}_{k}\right)+\eta_{k(t+1)}$

where $1=$ number of steps ahead (lead) in forecast and $\bar{z}_{k}=\sum_{j=1}^{J} k_{0, k j} X_{j(t+1-1)}$ (convergence to steady state).

But do you think you could have the forecast of short term variations of macroeconomic factors $\left(\Delta X_{j}(t+D)\right.$ in the long run? Are them available and robust?

\section{step6: guarantee coherence between lifetime PDs and stressed PDs}

The limited availability of long term economic forecasts along with their poor accuracy (economic forecasts are available for initial 3 years in EBA stress tests), implies a long term convergence hypothesis which is appropriate since default rate is hopefully stationary in mean.

We propose to neutralize the convergence process for the initial 3 years (in order to amplify the sensitivity of the models in short term exercises like stress test as required by regulators methodology) and to neutralize first differences in the next years in order to maximize long term convergence (as expected for IFRS9 purposes).

Formally,

$\left\{\begin{array}{c}\gamma=1 \quad \text { if } l=1,2,3 \ldots \text { years ahead in forecast } \\ \sum_{j=1}^{J} \beta_{0, k j} \Delta X_{j t+1}=0 \quad \text { if } l=4,5,6, \ldots, N \text { years ahead in forecast }\end{array}\right.$

Then: 


$$
\left\{\begin{array}{cl}
\Delta z_{k(t+1)}=\sum_{j=1}^{I} \beta_{0, k j} \Delta X_{j(t+1)}+\eta_{k(t+1)} & \text { if } l=1,2,3 \\
\Delta z_{k(t+1)}=(\gamma-1)\left(z_{k(t+1-1)}-\bar{z}_{k}\right)+\eta_{k(t+1)} & \text { if } l=4,5,6, \ldots, N
\end{array}\right.
$$

Actually, Stress testing exercises encourage the use of just the first expression with 3 years macroeconomic forecasts for stressed PD, whereas for IFRS 9 purposes both expressions are useful to derive long term $\Delta z$. The constraint of using only short term variations of macroeconomic factors is needed to avoid the generation of an unhampered process.

Several different assumptions could be suitable, but this hypothesis has the relevant feature that allows for the coherence between Stress Testing and IFRS 9 frameworks.

\section{step 7: forecast scenario dependent PDs and long-term PDs}

For "short term" exercises ( 3 years), given the assumption above, we can iteratively derive future PDs from the available PDs at time $t$, as follows:

$P D_{i(t+1)}=\Phi\left\lfloor\Phi^{-1}\left(P D_{i(t+1-1)}\right)-\frac{\alpha_{i}}{\sqrt{1-\alpha_{i}^{2}}} \sum_{j=1}^{J} \beta_{0, k j} \Delta X_{j(t+1)}\right\rfloor$

Under the assumption of macroeconomic variables' convergence (step5), we have that:

$z_{k(t+1)}=\gamma z_{k(t+1-1)}+(1-\gamma) \bar{z}_{k}$

For long term path we can express $P D_{\mathrm{i}(t+\mathbb{D}}$ as

$P D_{\mathrm{i}(t+1)}=\Phi\left[\gamma \Phi^{-1}\left(P D_{\mathrm{i}(t+1-1)}\right)+(1-\gamma) \Phi^{-1}\left(\overline{P D_{1}}\right)\right]$

Convergence pace to long term PD is governed by $\gamma$ which can be either defined to reach the long-run convergence in a specified time (e.g. we found that $y=0.5$ implies a convergence to the long-run in about 10 years), or estimated from the satellite model (see formulation in step5).

\section{step8: forecast scenario dependent and long-term migration probabilities}

As in step4, also the rating transition probabilities depend on $z$ factor, so the procedure to derive their path to the long term is analogous to PDs.

For short term exercises:

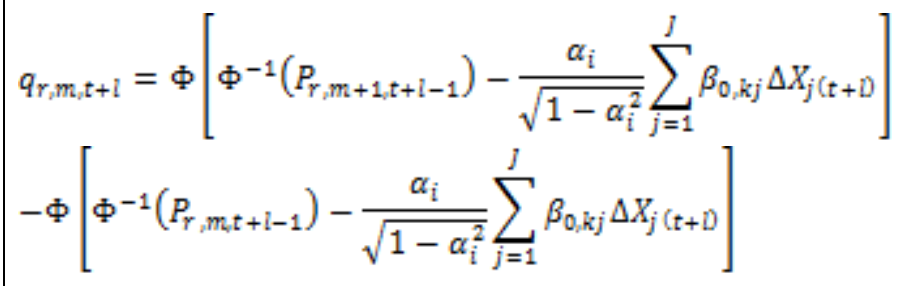

Whereas for long term path:

$$
\begin{aligned}
& q_{r_{2} m_{i}, t+1}=\Phi\left[\gamma \Phi^{-1}\left(P_{r_{2} m+1, t+1-1}\right)+(1-\gamma) \Phi^{-1}\left(\bar{P}_{r_{3} m+1}\right)\right] \\
& -\Phi\left[\gamma \Phi^{-1}\left(P_{r_{2} m_{2}, t+1-1}\right)+(1-\gamma) \Phi^{-1}\left(\bar{P}_{r_{3} m}\right)\right]
\end{aligned}
$$

\section{step9: derive scenario dependent lifetime PDs}

Once obtained the PIT scenario dependent PDs and migrations for each future year, it is easy to derive the forward looking cumulative PD curve by using an inhomogeneous Markov chain approach (see for example Suhov and Kelbert, 2008).

Generally speaking, according to the Markov approach, the scenario dependent cumulative transition matrix is given by the product of conditional transition matrices. 


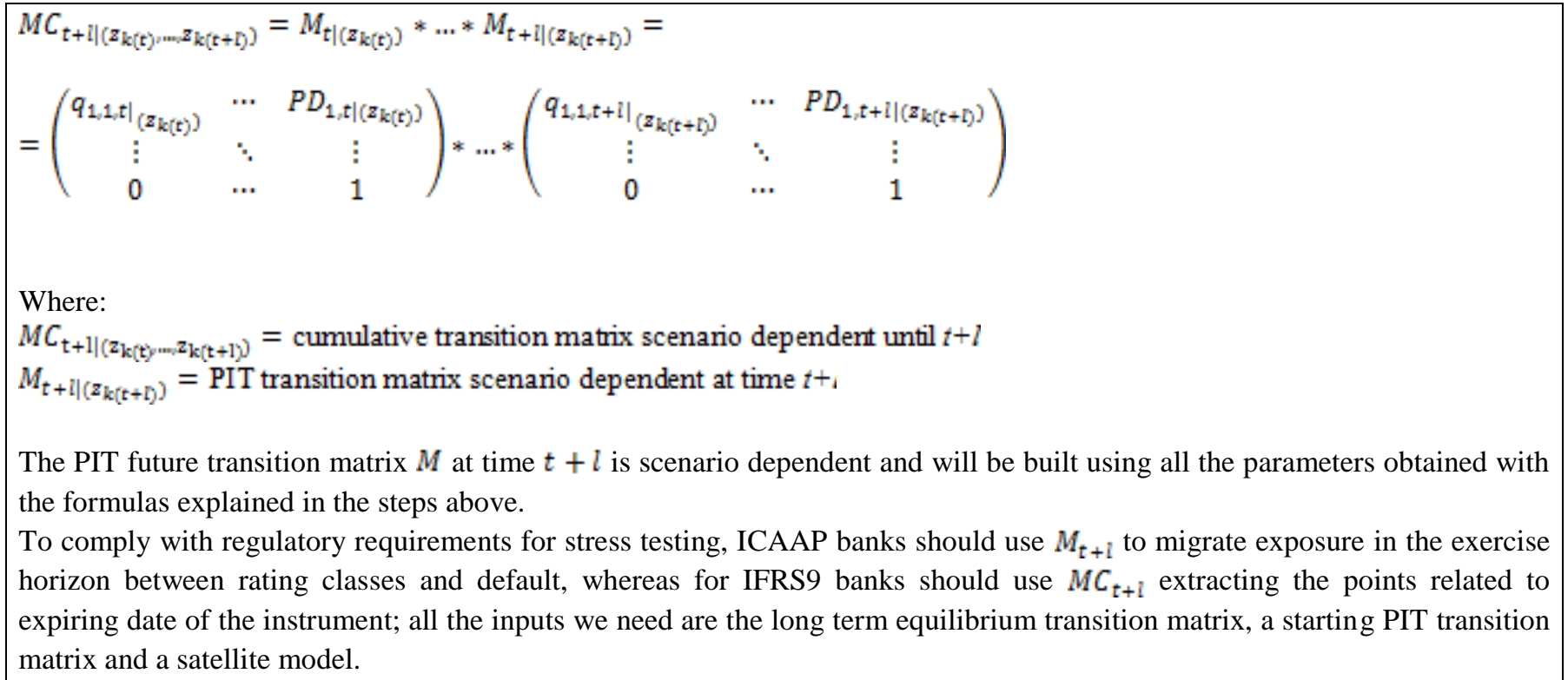

\section{step 10: assess the fitting of the estimated PD curves}

A relevant feature of the proposed methodology is the flexibility of the resulting PD curves with respect to variations in the convergence parameter $\gamma$ and in the long-term convergence matrix. This means that financial institutions could simply work on those values to maximize the estimated curves' fitting to the actual default curves.

However, even if we are aware that large part of financial institutions aim at maximizing PD curves' fitting, it is our opinion that such an objective is not actually needed, because of the regulatory purpose (see IFRS 9 regulation) of building cumulative curves which are forward looking and not backward looking. In other words, past default curves' shape should not be the target of the current forward looking modeling if the path implied into the future PIT matrices and the long term matrix defines different-shaped curves. Differences between the estimated curves and the past observed curves represent just the result of different expectations about the future macroeconomic scenario.

\section{Conclusions}

A comprehensive framework for PIT, stressed and lifetime PDs calculation was proposed in this paper. We have shown how banks could accomplish several different regulatory requirements through a unique framework promoting coherence between methodological frameworks, results and enhancing synergies in estimation work.

In particular, we have shown how banks could proceed by implementing the following 10 operational steps:

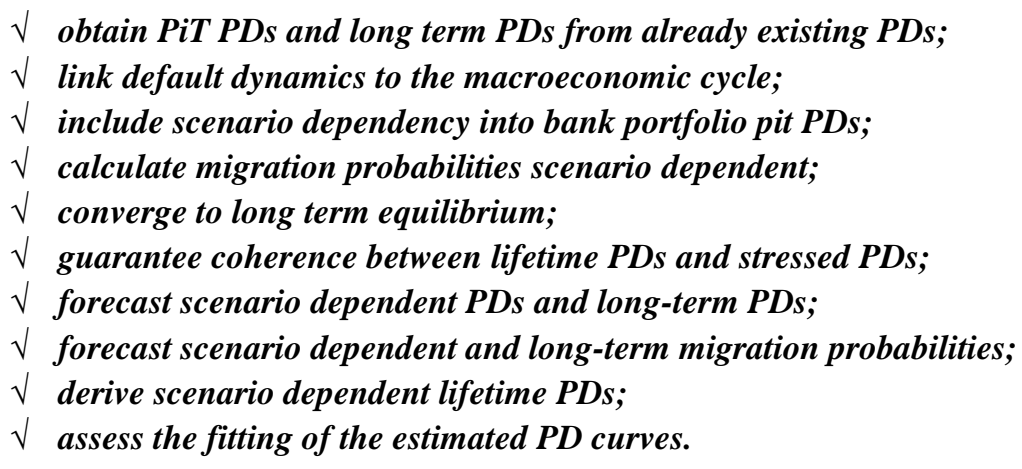

To comply with regulatory requirements for Stress Testing, ICAAP banks should implement steps that we called "short term" in this paper generating scenario dependency of PDs and migration matrices, whereas for IFRS9 compliance banks should implement all the steps described using also long term convergence.

We argue that such an approach provides further relevant features, such as the flexibility in the resulting output and a sound methodological basis.

We deem that a broad, comprehensive PD framework is becoming more and more necessary for an efficient steering of banks because of the increasing number of regulatory requirements. 
The proposed framework is sensitive to variations in the convergence speed parameter and to the convergence matrix, providing banks a flexible modeling tool able to produce the expected PD term structures. Moreover, this framework can build forward looking PD curves whose shape is not just the replication of past observed paths, but actually is the result of the expected macroeconomic scenario evolution (optimistic, baseline or adverse for example).

These considerations pave the way for the increasingly pressing theme of PD curves' backtesting. As we mentioned earlier, the proposed model (as well as any other PD term structure model developed for similar purposes) cannot be tested by simply comparing estimated PD curves with past observed results, it should instead be tested by taking into account the effect of the current and predicted macroeconomic scenario on large time horizons.

\section{Carlo Toffano, Francesco Nisi and Lorenzo Maurri}

\section{References}

1. BCBS, Guidance on accounting for expected credit losses, 2015.

2. Engle, R.; Granger, C. Co-integration and error correction. Econometrica, 55(2), 1987.

3. EBA, Final Report - Guidelines on ICAAP and ILAAP information collected for SREP purposes, 2016.

4. EBA, 2018 EU-Wide Stress Test - Draft methodological note, 2017.

5. European Parliament, Regulation no. 575/2013, 2013.

6. FSA, Use of variable scaling factors to derive long run probabilities of default for retail portfolios, 2007.

7. Merton, R.C. On the pricing of corporate debt: the risk structure of interest rates. Journal of Finance, $29,1974$.

8. Repullo, R., Saurina, J., and Trucharte, C. Mitigating the procyclicality of Basel II, CEMFI, 2009.

9. Rubtsov, M.; Petrov, A. A PIT-TTC approach to rating assignment and probability of default calibration. Journal of Risk Model Validation, 10(2), 2016.

10. Suhov, Y.; Kelbert, M. Markov Chains: A Primer in Random Processes and their Applications, CUP, 2008.

11. Van Gestel T., Credit stress testing. Proceedings of the CRC - Stress Testing Conference, 2014. Available at: https://www.business-school.ed.ac.uk/waf/mdb_event_v2/get_file.php?event_file_id=130.

12. Vasicek, O. Loan portfolio value. Risk, 15(12), 2002. 


\section{Lo Standardised Measurement Approach (SMA) per i rischi operativi}

di Giacomo Petrini (Banco BPM) e Camillo Giliberto (MPS) ${ }^{1}$

\section{ABSTRACT}

This contribution seeks to highlight in detail the implications of the proposed method both in terms of impacts on the requirement of own funds, and in terms of the evolution required in the underlying organizational processes. In particular, this work wants to emphasise how the new framework for calculating the requirement of own funds would reduce the risk sensitivity of the metrics used; The most evident consequences of this evolution would be on the current processes that use metrics for management purposes, in order to connect the measures of regulatory and economic capital. In this context, the evolution of the regulatory environment would result missing of opportunities that, currently, encourage the use of internal models for management purposes, so that the target proposed by the Committee could be achieved at the cost of an increase of systemic risk, resulting from the adoption of less risk sensitive techniques also for management purposes.

\section{Premessa}

Dopo la prima consultazione avviata nel mese di ottobre 2014, il Comitato di Basilea ha rilasciato il 4 marzo 2016 in seconda consultazione una nuova versione dello stesso documento "Standardised Measurement Approach for operational risk".

Il documento propone una evoluzione del quadro regolamentare di riferimento per la determinazione del requisito di fondi propri a fronte dei rischi operativi; in particolare, viene prospettato un nuovo metodo di misurazione e gestione dei rischi operativi (Standardised Measurement Approach, o SMA) che dovrebbe sostituire tutti gli approcci attualmente vigenti per il calcolo del requisito di fondi propri a fronte dei rischi operativi (Basic Indicator Approach, o BIA; Traditional Standardised Approach, o TSA; Advanced Measurement Approach, o AMA).

A tal fine, il framework proposto (SMA) si caratterizza per una differente metodologia di calcolo del requisito, che dovrebbe garantire una più agevole comparabilità delle risultanze, e per un insieme di criteri organizzativi minimi da applicare obbligatoriamente ai processi di identificazione, raccolta e trattamento dei dati di perdita operativa ${ }^{2}$.

D'altro canto, è intenzione del Regulator prevedere l'applicazione del framework SMA solo su base consolidata agli enti finanziari operanti a livello internazionale, ferma restando la possibilità (per i singoli supervisori nazionali) di prevederne l'applicazione anche alle banche operanti a livello nazionale.

Il presente contributo ha la finalità di evidenziare in modo dettagliato le implicazioni del metodo proposto sia in termini di impatti sul requisito di fondi propri, sia in termini di evoluzione richiesta nei processi organizzativi sottostanti. A tale scopo, nei paragrafi successivi si ripropongono in primis gli elementi che caratterizzano gli attuali metodi di regolamentazione (BIA, TSA, AMA); successivamente, si sintetizza la proposta di evoluzione regolamentare (metodo SMA), ponendo particolare attenzione ai suoi elementi costitutivi (la definizione di Business Indicator, o BI; il moltiplicatore delle perdite interne; la formula di calcolo del requisito di fondi propri; i requisiti minimi per l'utilizzo dei dati interni di perdita), anche in ottica comparata con analoghi elementi ad oggi utilizzati negli attuali metodi di regolamentazione; infine, si contestualizzano le principali considerazioni formulate dall'Associazione Bancaria Italiana (o ABI) in risposta alla consultazione, come formalizzate nel paper pubblicato in data 06 giugno 2016.

\section{L'attuale regolamentazione del requisito di fondi propri a fronte dei rischi operativi}

Il Regolamento (UE) n. 575/2013 del Parlamento europeo e del Consiglio del 26 giugno 2013 relativo ai requisiti prudenziali per gli enti creditizi e le imprese di investimento (d'ora in poi, CRR) definisce l'attuale contesto normativo di riferimento, tra gli altri, anche per la determinazione del requisito di fondi propri a fronte dei rischi operativi.

Il rischio operativo è definito come il rischio di perdite derivanti dalla inadeguatezza o dalla disfunzione di processi, risorse umane e sistemi interni, oppure da eventi esogeni, ivi compreso il rischio giuridico. ${ }^{3}$ I metodi esplicitati per la sua misurazione sono definiti all'interno di un contesto regolamentare complessivamente coeso, che muove dalla considerazione che il rischio operativo rappresenta un rischio notevole per gli enti, ed esso richiede pertanto una adeguata copertura con fondi propri; tenuto conto della diversità degli enti nell'Unione, vengono previsti sistemi alternativi di calcolo dei requisiti in materia di rischio operativo che incorporano livelli diversi di sensibilità al rischio e che richiedono gradi diversi di sofisticatezza, pur nella consapevolezza che occorra prevedere adeguati incentivi per spingere gli enti ad adottare i metodi che presentano una maggiore sensibilità al rischio. ${ }^{4}$ La stessa normativa riconosce in ogni caso che, essendo le tecniche di misurazione e di

${ }^{1}$ Le opinioni espresse dagli autori sono personali e non impegnano l'istituto di appartenenza.

${ }^{2}$ Cfr. par. 6.1, "General criteria on loss data identification, collection and treatment" e 6.2 "Specific criteria on loss data identification, collection and treatment" all'interno della sezione 6 "Minimum standards for the use of loss data under the SMA".

${ }^{3}$ Cfr. CRR, art. 3, par. 1, comma 52.

${ }^{4}$ Cfr. CRR, premettenda (52). 
gestione del rischio operativo in fase di continua evoluzione, occorre che le norme siano soggette a costante riesame e se del caso aggiornate. ${ }^{5}$

I requisiti di dettaglio definiti per i diversi metodi di misurazione allo stato previsti sono descritti negli artt. $312-324$ della CRR e vengono di seguito brevemente richiamati per gli apsetti rilevanti ai fini di una valutazione compiuta del metodo SMA.

\subsection{Il metodo BIA}

Per le banche che decidono di applicare il metodo BIA, il requisito di fondi propri a fronte dei rischi operativi è pari al $15 \%$ della media triennale dell'indicatore rilevante; esso è dunque calcolato sulla base delle tre ultime osservazioni su base annuale effettuate alla fine dell'esercizio; qualora da una delle osservazioni risulti che l'indicatore rilevante è negativo o nullo, le banche non tengono conto di questo dato nel calcolo della media triennale: la media triennale è calcolata come la somma dei dati positivi divisa per il numero dei dati positivi.

L'indicatore rilevante è definito puntualmente dalla normativa di riferimento ${ }^{6}$ come somma algebrica (ottenuta includendo ciascun elemento nella somma con il suo segno positivo o negativo) di:

2) Interessi e proventi assimilati;

3) Interessi e oneri assimilati;

4) Proventi su azioni, quote ed altri titoli a reddito variabile/fisso;

5) Proventi per commissioni/provvigioni;

6) Oneri per commissioni/provvigioni;

7) Profitto (perdita) da operazioni finanziarie;

8) Altri proventi di gestione.

Le banche devono correggere i predetti elementi tenendo conto dei seguenti requisiti:

- l'indicatore rilevante va calcolato al lordo di accantonamenti e di spese operative; nelle spese operative devono essere incluse le provvigioni versate per i servizi forniti in outsourcing da terzi che non sono l'impresa madre o filiazioni della banca né filiazioni di un'impresa madre che è anche l'impresa madre della banca; le banche possono utilizzare le spese per i servizi forniti in outsourcing da terzi per ridurre l'indicatore rilevante soltanto se sostenute da un'impresa sottoposta a vigilanza bancaria;

- $\quad$ nel calcolo dell'indicatore rilevante, le banche non possono utilizzare i seguenti elementi:

- profitti e perdite realizzati sulla vendita di titoli non inclusi nel portafoglio di negoziazione,

- i proventi derivanti da partite straordinarie o irregolari,

- i proventi derivanti da assicurazioni;

- quando la rivalutazione di titoli del portafoglio di negoziazione rientra nel conto profitti e perdite, le banche possono includere tale rivalutazione.

\subsection{Il metodo TSA}

Per poter utilizzare il metodo TSA, le banche devono soddisfare una serie di requisiti organizzativi puntualmente elencati: ${ }^{7}$

1) disporre di un sistema di gestione e di valutazione del rischio operativo ben documentato e con responsabilità chiaramente assegnate; detto sistema deve rilevare le esposizioni della banca soggette al rischio operativo e raccogliere i dati rilevanti sul rischio operativo, incluse le perdite significative. Questo sistema deve essere assoggettato a revisioni periodiche indipendenti svolte da un soggetto interno od esterno dotato delle competenze necessarie a tal fine;

2) integrare strettamente il sistema di valutazione del rischio operativo nei processi di gestione del rischio della banca. I risultati prodotti da tale sistema devono costituire parte integrante del processo di sorveglianza e controllo del profilo di rischio operativo dell'ente;

3) disporre di un sistema di reportistica all'alta dirigenza che fornisca comunicazioni sull'esposizione al rischio operativo ai responsabili delle funzioni rilevanti all'interno della banca; la banca deve anche dotarsi di procedure per intraprendere azioni appropriate sulla base delle informazioni contenute in tali comunicazioni.

Ove tali requisiti siano soddisfatti, la banca può adottare il metodo TSA per il calcolo del requisito di fondi propri a fronte del rischio operativo previa comunicazione preventiva all'Autorità di Vigilanza competente.

L'utilizzo del metodo TSA richiede alla banca di suddividere le proprie attività in otto linee di business ${ }^{8}$, elaborando politiche e criteri specifici documentati per ripartire l'indicatore rilevante tra le linee di business; le politiche e i criteri vanno rivisti ed adattati, se del caso, in funzione di attività e rischi nuovi o mutevoli.

Il requisito di fondi propri a fronte dei rischi operativi si ottiene, in questo caso, come la media triennale della somma dei requisiti annuali di fondi propri per tutte le suddette linee di business; il requisito annuale in materia di fondi propri per ogni linea di business è pari al prodotto della percentuale di ponderazione prevista dalla normativa (c.d. fattore beta $)^{9}$ e della parte dell'indicatore rilevante classificata nella linea di business interessata. Il calcolo della media triennale è effettuato in ogni caso sulla base delle tre ultime osservazioni su base annuale effettuate alla fine dell'esercizio.

\footnotetext{
${ }^{5}$ Cfr. ibidem.

${ }^{6}$ Cfr. CRR, art. 316.

${ }^{7}$ Cfr. CRR, art. 320 .

${ }^{8}$ Cfr. CRR, art. 317, par. 4, tabella 2 e CRR art. 318, par. 2 per I principi di classificazione.

${ }^{9}$ Cfr. CRR, art. 317, par. 4, tabella 2, colonna 3.
} 
In aggiunta, per ogni anno, gli enti possono compensare senza limiti i requisiti negativi di fondi propri dovuti alla parte negativa dell'indicatore rilevante di una linea di business con i requisiti positivi in altre linee di business; tuttavia, qualora i requisiti aggregati di fondi propri di tutte le linee di business in un determinato anno siano negativi, per quell'anno l'ente immette al numeratore il valore zero.

Esiste inoltre la possibilità, pure prevista dalla normativa di riferimento ${ }^{10}$, di utilizzare il c.d. metodo standardizzato alternativo (alternative standardised approach, o ASA), nel quale (per le sole linee di business "servizi bancari al dettaglio" e "servizi bancari a carattere commerciale") le banche possono applicare, come misura dell'indicatore rilevante, un indicatore di reddito normalizzato pari all'ammontare nominale dei crediti e degli anticipi moltiplicato per 0,035; in tal caso, i crediti e gli anticipi sono composti dagli importi complessivamente utilizzati nei corrispondenti portafogli creditizi. Inolte, per la linea di business "servizi bancari a carattere commerciale", gli enti possono includere (nell'ammontare nominale dei crediti e degli anticipi) anche i titoli non detenuti nel portafoglio di negoziazione.

L'utilizzo del metodo ASA è soggetto ad autorizzazione da parte dell'Autorità di Vigilanza competente, e richiede che siano soddisfatte tutte le seguenti condizioni:

a) i servizi bancari al dettaglio o i servizi bancari a carattere commerciale devono costituire almeno il $90 \%$ del reddito complessivo;

b) una quota significativa dei servizi bancari al dettaglio o dei servizi bancari a carattere commerciale deve includere prestiti associati ad un'elevata PD;

c) il metodo ASA deve fornire una base appropriata per il calcolo del requisito di fondi propri per il rischio operativo.

\subsection{I metodi AMA}

L'utilizzo dei metodi avanzati di misurazione del requisito patrimoniale a fronte dei rischi operativi (o metodi AMA) è soggetto ad autorizzazione da parte dell'Autorità di Vigilanza competente: l'autorizzazione ad impiegare detti metodi, basati su sistemi di misurazione del rischio operativo sviluppati internamente dalla banca, richiede che siano interamente soddisfatti tutti i requisiti organizzativi (qualitativi e quantitativi) puntualmente elencati dalla $\mathrm{CRR}^{11}$ e di seguito riportati per completezza:

1) Requisiti qualitativi:

1.1 il sistema interno di misurazione del rischio operativo della banca deve essere strettamente integrato nei processi di gestione quotidiana del rischio;

1.2 la banca deve disporre di una funzione indipendente di gestione del rischio operativo;

1.3 la banca deve dotarsi di strumenti di segnalazione periodica delle esposizioni al rischio operativo e delle perdite rilevate e di procedure per intraprendere appropriate azioni correttive;

1.4 il sistema di gestione del rischio della banca deve essere ben documentato; la banca deve disporre di procedure che assicurino l'osservanza dei requisiti e deve prevedere direttive per il trattamento dei casi di difformità;

1.5 la banca deve sottoporre i processi di gestione del rischio operativo e i relativi sistemi di misurazione a revisioni periodiche effettuate da revisori interni o esterni;

1.6 i processi interni di validazione devono operare in maniera corretta ed efficace;

1.7 i flussi di dati e i processi associati al sistema di misurazione del rischio dell'ente devono essere trasparenti e accessibili.

2) Requisiti quantitativi (che includono requisiti relativi al processo, ai dati interni, ai dati esterni, all'analisi di scenario, ai fattori del contesto operativo e del sistema dei controlli interni):

2.1 Requisiti quantitativi relativi al processo:

2.1.1 la banca deve calcolare il proprio requisito di fondi propri come somma delle perdite attese e di quelle inattese, a meno che le perdite attese non siano adeguatamente stimate nelle prassi operative interne. Il metodo di misurazione del rischio operativo deve essere in grado di cogliere potenziali eventi di perdita a elevato impatto, raggiungendo standard di robustezza comparabili a quelli di un intervallo di confidenza del 99,9\% su un periodo di un anno;

2.1.2 il sistema di misurazione del rischio operativo deve comprendere comprende l'impiego di dati interni e di dati esterni, le analisi di scenario e i fattori che rispecchiano il contesto operativo e il sistema dei controlli interni; la banca deve dotarsi di un metodo ben documentato per ponderare l'uso di questi quattro elementi nel suo sistema complessivo di misurazione del rischio operativo;

2.1.3 il sistema di misurazione del rischio dell'ente deve cogliere le determinanti principali del rischio che influiscono sul profilo della coda della distribuzione stimata delle perdite;

2.1.4 la banca può prendere in considerazione le correlazioni relative alle perdite per rischio operativo tra le singole stime del rischio operativo soltanto se i suoi sistemi per la misurazione delle correlazioni sono solidi e applicati con correttezza e tengono conto dell'incertezza associata a stime di questo tipo, specialmente in periodi di stress; in yal caso, la banca deve validare le proprie ipotesi sulle correlazioni attraverso appropriate tecniche quantitative e qualitative;

${ }^{10}$ Cfr. CRR, art. 319.

${ }^{1}$ Cfr. CRR, art. 321-322. 
2.1.5 il sistema di misurazione del rischio dell'ente deve essere intrinsecamente coerente ed evitare duplicazioni nel computo delle valutazioni qualitative o delle tecniche di attenuazione del rischio riconosciute in altre disposizioni della CRR.

2.2 Requisiti relativi ai dati interni:

2.2.1 la banca deve basare le proprie misurazioni interne del rischio operativo su un periodo di osservazione di almeno cinque anni; se la banca adotta per la prima volta i metodi AMA, si può utilizzare un periodo di osservazione di tre anni;

2.2.2 la banca deve essere in grado di classificare i propri dati storici sulle perdite in funzione delle pertinenti linee di business definite per il metodo TSA e delle tipologie di eventi definite dalla normativa di riferimento ${ }^{12}$, nonché di fornire su richiesta tali dati alle Autorità competenti; in circostanze eccezionali la banca può attribuire gli eventi di perdita che interessano l'intera istituzione ad una linea di business aggiuntiva, denominata "elementi d'impresa". La banca deve in ogni caso dotarsi di criteri documentati e oggettivi per attribuire le perdite alle specifiche linee di business e tipologie di eventi. In ogni caso, la banca deve registrare nella banca dati sul rischio operativo, e rilevare separatamente, le perdite da rischio operativo collegate al rischio di credito, storicamente incluse nella banca dati interna relativa al rischio di credito: tali perdite non sono soggette all'applicazione del requisito previsto per il rischio operativo a condizione che la banca sia tenuta a continuare a trattarle come rischio di credito ai fini del calcolo dei requisiti di fondi propri. L'ente deve infine includere le perdite da rischio operativo collegate ai rischi di mercato nel calcolo del requisito di fondi propri per il rischio operativo;

2.2.3 i dati interni sulle perdite della banca devono essere esaurienti, nel senso che devono cogliere tutte le attività e le esposizioni rilevanti da tutti i pertinenti sottosistemi e articolazioni territoriali; la banca deve essere inoltre in grado di dimostrare che l'eventuale esclusione di attività o di esposizioni, individualmente o in combinazione tra loro, non produca un impatto significativo sulle stime di rischio complessive. La banca deve poi definire adeguate soglie minime di perdita per la raccolta dei dati interni;

2.2.4 oltre ai dati sugli importi della perdita lorda, la banca deve raccogliere informazioni sulla data dell'evento, su eventuali recuperi degli importi lordi, nonché informazioni descrittive sulle determinanti o sulle cause dell'evento di perdita;

2.2.5 la banca deve dotarsi di criteri specifici per classificare i dati relativi alle perdite derivanti da un determinato evento di perdita verificatosi in una funzione centralizzata ovvero in un'attività che si estenda su più linee di business, come pure da eventi collegati tra loro nel tempo;

2.2.6 la banca deve disporre di procedure documentate per valutare la rilevanza su base continuativa dei dati storici sulle perdite, compresi i casi in cui si possa ricorrere a rettifiche discrezionali, riparametrazioni o altri aggiustamenti, in quale misura essi possono essere effettuati e quali debbano essere i responsabili di tali decisioni.

2.3 Requisiti di idoneità relativi ai dati esterni:

2.3.1 il sistema di misurazione del rischio operativo della banca deve utilizzare dati esterni pertinenti, specialmente quando vi è motivo di ritenere che la banca sia esposta a perdite a impatto potenzialmente elevato, ancorché infrequenti; la banca deve disporre di un processo sistematico atto a individuare le situazioni in cui impiegare i dati esterni e le metodologie usate per incorporare tali dati nel proprio sistema di misurazione;

2.3.2 la banca deve rivedere regolarmente le condizioni e le prassi per l'utilizzo di dati esterni, documentarle ed assoggettarle a periodica revisione indipendente.

2.4 Requisiti relativi alle analisi di scenario (per valutare la propria esposizione a eventi di particolare gravità, la banca deve infatti utilizzare, unitamente ai dati esterni, analisi di scenario condotte da esperti):

2.4.1 per garantirne la fondatezza, le valutazioni incluse nelle analisi di scenario devono essere nel tempo validate e rivedute in base al confronto con le perdite effettivamente subite.

2.5 Requisiti di idoneità relativi ai fattori del contesto operativo e del sistema dei controlli interni:

2.5.1 la banca deve dotarsi di una metodologia complessiva di valutazione del rischio capace di cogliere i fattori cruciali del contesto operativo e del sistema dei controlli interni, che possono modificare il profilo di rischio operativo dell'ente stesso;

2.5.2 la banca deve giustificare la scelta di ciascun fattore tenendo conto della significatività dello stesso quale determinante del rischio, sulla base dell'esperienza e del giudizio degli esperti delle aree di business interessate;

2.5.3 la banca deve essere in grado di dimostrare alle Autorità competenti la sensibilità delle stime di rischio ai mutamenti dei fattori e la ponderazione relativa dei vari fattori; oltre a cogliere le modifiche del profilo di rischio dovute al miglioramento dei sistemi di controllo, il sistema di misurazione del rischio della banca deve infatti individuare anche potenziali aumenti del rischio derivanti dalla maggiore complessità delle attività o dagli accresciuti volumi operativi;

${ }^{12}$ Cfr. CRR, art. 324. 
2.5.4 la banca deve documentare il proprio sistema di misurazione del rischio e sottoporlo a revisione indipendente interna e da parte delle autorità competenti; inoltre, nel corso del tempo, la banca deve validare e rivedere il processo e i risultati mediante il confronto con le perdite interne effettivamente subite e dati esterni pertinenti.

Stante il rispetto di tutti i suddetti requisiti, l'utilizzo dei metodi AMA consente alla banca (previa autorizzazione delle Autorità di Vigilanza competenti) di riconoscere l'effetto delle assicurazioni e di altri meccanismi di trasferimento del rischio ove la banca possa dimostrare il conseguimento di un significativo effetto di attenuazione del rischio; tale effetto non può in ogni caso superare il $20 \%$ del requisito di fondi propri a fronte del rischio operativo precedente al riconoscimento delle tecniche di attenuazione del rischio.

Detto riconoscimento è soggetto al rispetto di una ulteriore serie di requisiti, puntualmente elencati dalla normativa di riferimento $^{13}$, relativi all'assicuratore (merito di credito, indipendenza giuridica dalla banca garantita), alle caratteristiche delle polizze (durata iniziale, durata residua, periodo minimo di preavviso di disdetta, assenza di escape clauses) e alla metodologia con cui esse sono incluse nel modello di calcolo del requisito a fini di attenuazione del rischio. ${ }^{14}$

\section{Il metodo SMA - Una introduzione}

Nel mese di ottobre 2014, il Comitato di Basilea ha proposto una prima revisione dei metodi TSA e BIA per la misurazione del requisito di fondi propri a fronte dei rischi operativi, allo scopo di fornire chiare indicazioni per il superamento delle rispettive debolezze nel frattempo emerse; contestualmente, lo stesso organo ha avviato anche una complessiva rivisitazione di costi e benefici connessi all'adozione dei metodi AMA.

Tale processo si è concluso nel marzo 2016, allorché il Comitato di Basilea ha completato la seconda versione del documento "Standardised Measurement Approach for operational risk", poi rilasciato per la consultazione nel successivo mese di giugno; tale documento propone ulteriori revisioni del quadro normativo, che hanno come principale obiettivo quello di bilanciare in modo più efficace la semplicità, la trasparenza e la sensitivity al rischio dei metodi di calcolo del requisito di fondi propri a fronte dei rischi operativi.

In particolare, il documento consegue dall'azione intrapresa dal Comitato nel biennio 2014-2016, volta ad indirizzare i temi della marcata variabilità osservata negli Attivi Ponderati per il Rischio (Risk Weighted Assets, o RWA) tra le istituzioni bancarie di diverse giurisdizioni, nonché della ridotta comparabilità degli stessi in relazione alle diverse tipologie di rischio (mercato, credito e controparte, operativo).

Avendo individuato la causa principale della suddetta variabilità degli RWA nelle diverse declinazioni degli approcci metodologici di calcolo, il Comitato ha avviato una complessiva azione di semplificazione degli stessi approcci, volta a mantenerne la sensitivity ai diversi rischi sottostanti pur in un contesto di maggiore oggettività e trasparenza metodologica; con specifico riferimento ai rischi operativi, la direzione intrapresa si traduce quindi nella definizione di una nuova metodologia (il metodo SMA), nella quale la combinazione di alcuni elementi chiave del metodo TSA, unitamente all'esperienza rappresentata dai dati interni di perdita, consente di ricavare una nuova misura di esposizione ai rischi operativi che viene in ogni caso ritenuta ancora sufficientemente risk sensitive. Il Comitato reputa inoltre che questa nuova misura (esplicitamente presentata come non-model-based) preservi la semplicità e la comparabilità dei conseguenti requisiti di fondi propri, propria dei metodi TSA e BIA, consentendo congiuntamente di ridurre la complessità dei modelli sottostanti.

L'analisi proposta dal Comitato, dalle cui risultanze il nuovo metodo SMA viene elaborato, evidenzia infatti le problematiche connesse all'adozione dei metodi AMA, basati su un insieme variegato di metodologie di modellazione interna, pur soggette ad approvazione regolamentare: infatti la flessibilità originariamente prevista per tali metodi, che derivava dalla mancanza di paradigmi strutturati per la misurazione dei rischi operativi, avrebbe dovuto nel tempo ridursi con il convergere delle metodologie impiegate verso le c.d. best practice di settore.

In realtà, le analisi svolte dal Comitato, sui modelli di misurazione effettivamente realizzati dal sistema bancario e sulle conseguenti misure di requisito di fondi propri. hanno mostrato che il suddetto processo di progressiva convergenza non si è realizzato: la complessità intrinseca dei metodi AMA, unitamente alla difficile comparabilità dei loro esiti in termini di requisito (derivante anche dalla pluralità di modelli impiegati), hanno accresciuto la volatilità dei calcoli degli RWA, minacciando di ridurne l'attendibilità; per tale ragione, il Comitato ritiene che non vi sia più spazio per i modelli interni nel calcolo del requisito di fondi propri per i rischi operativi, e che sia opportuna l'adozione di un approccio più simile ai precedenti metodi TSA e BIA.

Il Comitato evidenzia peraltro che l'obiettivo del metodo SMA non è quello di accrescere in modo significativo il requisito di fondi propri per il rischio operativo, sebbene tale effetto (pur variabile da banca a banca) possa in alcuni casi realizzarsi.

Il nuovo metodo (di derivazione tipicamente attuariale) prevede l'utilizzo congiunto di dati contabili (per la determinazione del c.d. Business Indicator, o BI) e di dati interni relativi alle perdite storicamente registrate (per la determinazione della c.d. Loss Component, o LC), al fine di determinare un requisito di fondi propri che risulti proporzionale sia alla dimensione del volume di affari che all'ammontare delle perdite operative storicamente registrate; il presupposto sottostante è che

\footnotetext{
${ }^{13}$ Cfr. CRR, art. 323.
}

${ }^{14}$ Per completezza, si osserva che, in aggiunta a quanto prescritto dall'art. 446 della CRR, l'art. 454 prescrive anche che "Gli enti che utilizzano i metodi avanzati di misurazione di cui agli articoli da 321 a 324 per il calcolo dei requisiti di fondi propri per il rischio operativo pubblicano una descrizione dell'uso delle assicurazioni e di altri meccanismi di trasferimento del rischio ai fini dell'attenuazione del rischio" all'interno dell'informativa fornita al pubblico. 
l'esposizione per perdite operative cresce in modo più che proporzionale rispetto al BI, ed il calcolo di quest'ultimo attraverso dati di bilancio lo rende direttamente confrontabile tra le diverse banche.

D'altro canto, l'utilizzo dei dati interni relativi alle perdite storicamente registrate introduce un incentivo esplicito ad accrescere l'efficacia del sistema di gestione dei rischi operativi, dato che quanto più efficace risulta detto sistema, tanto più ridotta è l'esposizione alle perdite operative, anche a parità di BI.

Nei paragrafi successivi, vengono sintetizzate le caratteristiche principali del metodo SMA, come proposto nell'ultimo documento di consultazione "Standardised Measurement Approach for operational risk" del Comitato di Basilea.

\section{Il Business Indicator e la Business Indicator Component}

Il Business Indicator BI è pari alla somma dei valori assoluti (dunque, considerati con segno positivo) di un insieme significativo degli elementi del Conto Economico, che compongono il margine d'intermediazione lordo (o Gross Income), nonché di altre componenti reddituali collegate ad attività che producono rischi operativi, di solito escluse dal Gross Income (ad esempio, le componenti del P\&L del banking book) o portate in compensazione (ad esempio, commissioni passive ed altre spese operative).

Le componenti vengono tutte considerate in valore assoluto allo scopo di rappresentare il complessivo volume d'affari della banca, indipendentemente dalla eventuale compensazione delle singole voci, cosicché l'aggregato risultante risulta coerente con l'obiettivo della proposta del Comitato.

Nel documento di consultazione di marzo 2016, il Comitato ha adottato una versione rivista del Business Indicator (rispetto alla versione originariamente proposta nel 2014), al fine di risolvere le problematiche relative:

a) al differente trattamento che altrimenti avrebbero ricevuto i modelli di business "distribute only" e "originate to distribute": nella versione originaria del BI, infatti, le banche che distribuiscono prodotti acquistati da terzi dovevano includere nel BI sia i proventi di vendita che le spese di acquisto, mentre le banche che producono internamente $\mathrm{i}$ prodotti da distribuire dovevano includere nel BI solo i proventi di vendita;

b) alla componente del reddito distribuito attraverso i dividendi, il cui trattamento contabile differisce da paese a paese;

c) al tendenziale eccesso di requisito che sarebbe stato altrimenti richiesto alle banche con più elevato margine di interesse netto, indipendentemente dai rischi operativi sottostanti;

d) al tendenziale eccesso di requisito che sarebbe stato altrimenti richiesto alle banche con più alti livelli di commissioni passive o di spese operative (di nuovo, indipendentemente dai rischi operativi sottostanti);

e) all'equiparazione delle operazioni di leasing alle operazioni creditizie.

Il Business Indicator (BI) viene dunque definito ora come la somma di tre distinte componenti, ciascuna determinata come media dei valori sottostanti riferiti all'ultimo triennio:

Nella [1]:

$$
B I=I L D C_{A v g}+S C_{A v g}+F C_{A v g}
$$

- Avg indica che ciascuna componente è calcolata come media triennale degli anni t, t-1 e t-2;

- $\quad I L D C_{A v g}$ rappresenta la componente che include i redditi operativi netti derivanti da interessi o da operazioni di leasing, ovvero distribuiti in forma di dividendi:

$$
I L D C_{A v g}=\operatorname{Min}\left[A b s\left(I I_{A v g}-I E_{A v g}\right) ; 0,035 * I E A_{A v g}\right]+A b s\left(L I_{A v g}-L E_{A v g}\right)+D I_{A v g}
$$

Nella [2] viene adottata una normalizzazione per le banche con un alto margine di interesse netto (NIM), qualora esso sia superiore al 3,5\%: in questo secondo caso, infatti, la componente interessi viene regolata dal cap sul NIM, posto pari al 3,5\%; inoltre, data l'equiparazione delle operazioni di leasing alle operazioni creditizie, il reddito e le spese (compresi gli ammortamenti) derivanti da operazioni di leasing sono tra loro compensati, ed il valore netto compensato viene incluso in valore assoluto all'interno della componente in esame;

- $\quad S C_{A v g}$ rappresenta la componente che misura l'operatività generata dai servizi: essa è calcolata nel modo seguente: Max (Fee Income; Fee Expense) + Max (Other Operating Income; Other Operating Expense). La nuova modalità di calcolo migliora la risk sensitive rispetto agli attuali approcci, in quanto le parti relative a "Fee" e "Other Operating" non vengono considerate al netto delle relative spese; pertanto le banche con un elevato volume di business da servizi, ma con un basso margine, vengono trattate in modo diverso dalle banche con un piccolo volume di business da servizi:

$$
\begin{aligned}
S C_{A v g}= & \operatorname{Max}\left(O O I_{A v g} ; O O E_{A v g}\right)+\operatorname{Max}\left\{A b s\left(F I_{A v g}-F E_{A v g}\right) ; \operatorname{Min}\left[\operatorname{Max}\left(F I_{A v g} ; F E_{A v g}\right) ;\right.\right. \\
& \left.\left.0,5 * u B I+0,1 *\left(\operatorname{Max}\left(F I_{A v g} ; F E_{A v g}\right)-0,5 * u B I\right)\right]\right\}
\end{aligned}
$$

essendo: 
In questo caso, ai fini del calcolo della componente $S C_{A v g}$, nel caso di commissioni passive superiori al $50 \%$ del BI non rettificato, l'indicatore è incrementato solo per un importo pari al 10\% dell'eccedenza, con il valore assoluto delle commissioni nette che viene assunto come floor per evitare indesiderate riduzioni di capitale;

- $F C_{A v g}$ rappresenta la componente finanziaria, che include i redditi operativi netti derivanti da servizi finanziari:

$$
F C_{A v g}=A b s\left(N e t P \& L T B_{A v g}\right)+A b s\left(N e t P \& L B B_{A v g}\right)
$$

La Tavola 1, di seguito riportata, riepiloga le abbreviazioni utilizzate nel calcolo:

Tav. 1: Lista delle abbreviazioni (in ordine alfabetico)

\begin{tabular}{|c|l}
\hline$A b s$ & Valore assoluto dell'elemento incluso tra le successive parentesi \\
$B B$ & Banking Book \\
$B I$ & Business Indicator \\
\hline$D I$ & Redditi operativi netti distribuiti in forma di dividendi \\
$F C$ & Componente finanziaria \\
\hline$E A$ & Interest Earning Assets \\
\hline$I E$ & Spese per interessi (al netto di quelle per operazioni di leasing finanziario o operativo) \\
$I I$ & Reddito da interessi (al netto di quello derivante da operazioni di leasing finanziario o \\
& operativo) \\
\hline$I L D C$ & Componente che include redditi operativi netti derivanti da interessi o da operazioni di leasing, \\
& ovvero distribuiti in forma di dividendi \\
\hline$L E$ & Spese derivanti da operazioni di leasing (compresi gli ammortamenti) \\
\hline$L I$ & Reddito derivante da operazioni di leasing \\
$M a x$ & Valore massimo tra gli elementi inclusi tra le successive parentesi \\
\hline$M i n$ & Valore minimo tra gli elementi inclusi tra le successive parentesi \\
$O O E$ & Altre spese operative \\
$O O I$ & Altro reddito operativo \\
\hline$P L$ & Profit \& Loss \\
\hline$S C$ & Componente che misura l'operatività generata dai servizi \\
\hline$T B$ & Trading Book \\
\hline$U B I$ & BI non rettificato (cioè non aggiustato per elevaye commissioni passive) \\
\hline
\end{tabular}

Partendo dal Business Indicator BI, viene ricavata la Business Indicator Component, che rappresenta il livello medio delle perdite operative corrispondenti al dato livello di BI: questa trasformazione (cfr. Tav. 2) avviene categorizzando il BI in buckets, con coefficienti marginali progressivamente crescenti, sulla base dei dati di settore ricavati dal QIS condotto dal Comitato nel corso del 2015:

Tav. 2: Buckets di BI e quantificazione della BI component

\begin{tabular}{c|c|c}
\hline Bucket di BI & Range di BI & BI component \\
\hline 1 & da $€ 0$ a 1 bln. & $0,11^{*} \mathrm{BI}$ \\
2 & da $€ 1$ bln. $\mathrm{a} € 3 \mathrm{bln}$. & $€ 110 \mathrm{mln} .+0,15^{*}(\mathrm{BI}-€ 1 \mathrm{bln})$. \\
3 & da $€ 3 \mathrm{bln} . \mathrm{a} € 10 \mathrm{bln}$. & $€ 410 \mathrm{mln} .+0,19^{*}(\mathrm{BI}-€ 3 \mathrm{bln})$. \\
4 & da $€ 10 \mathrm{bln} . \mathrm{a} € 30 \mathrm{bln}$. & $€ 1,74 \mathrm{bln} .+0,23 *(\mathrm{BI}-€ 10 \mathrm{bln})$. \\
\hline 5 & oltre $€ 30 \mathrm{bln}$. & $€ 6,34 \mathrm{bln} .+0,29 *(\mathrm{BI}-€ 30 \mathrm{bln})$. \\
\hline
\end{tabular}

Nel Grafico 1 di seguito riportato, si rappresenta il valore della BI component (in ordinata) come funzione del valore di BI (espresso in ascissa); entrambe le scale adottate sono espresse in $€$ bln. La rappresentazione grafica evidenzia in modo chiaro come la BI component cresca più che proporzionalmente rispetto al BI, per effetto dei coefficienti marginali crescenti applicati (cfr. Tav. 2); ne consegue infatti che l'effetto marginale del BI sul BI component è maggiore per i buckets superiori rispetto a quelli inferiori. 


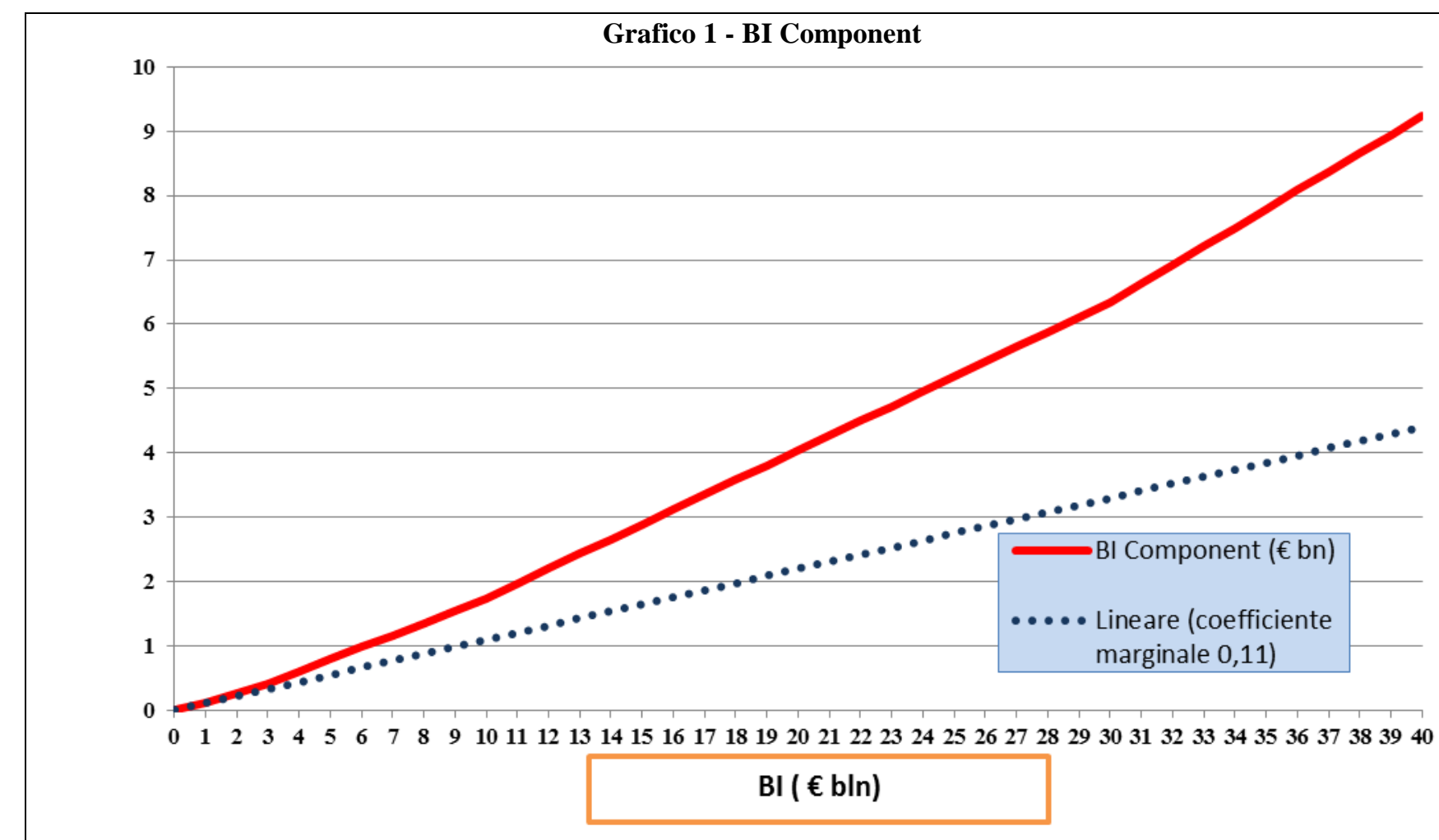

\section{La Loss Component e il moltiplicatore delle Internal Losses}

Il metodo SMA si fonda sul presupposto che il rapporto tra il Business Indicator BI e l'esposizione alle perdite operative sia relativamente simile per banche che presentano valori simili di BI. E' tuttavia possibile riscontrare differenti importi totali di perdite operative anche tra banche che presentano valor simili di BI; a tal fine, il metodo di calcolo del requisito di fondi propri, previsto dal metodo SMA, incorpora anche un fattore che tiene conto delle effettive perdite operative registrate storicamente da ciascuna banca (c.d. Loss component): tale inclusione consente, da un lato, di accrescere la risk sensitivity del requisito, mentre dall'altro fornisce un incentivo alle banche per migliorare i processi interni di gestione dei rischi operativi.

Il Comitato constata che le banche che utilizzano gli attuali metodi AMA e TSA (compresa la versione dell'Alternative Standardised Approach, o ASA) già raccolgono i dati sulle perdite operative interne, per cui esse sono già in grado di calcolare la componente storica delle perdite previste dal nuovo metodo SMA: in particolare, i risultati del QIS mostrano che più dell' $80 \%$ delle banche partecipanti, con BI $>€ 1$ bln., utilizzano già approcci diversi dal BIA, mentre solo il restante 20\% utilizza il BIA. Per questo motivo, il Comitato prospetta che la Loss Component sia utilizzata solo dalle banche con BI maggiore di $€ 1$ bln., e quindi non dalle banche ricomprese nel bucket 1della Tav. 2.

Per tutte le banche incluse nei bucket 2, 3, 4 e 5 della Tav. 2, quindi, il Comitato propone l'adozione di una misura che tenga conto delle effettive perdite operative registrate storicamente da ciascuna banca (c.d. Loss Component), da calcolarsi (come media dei dati dell'ultimo decennio) sulla base della seguente formula:

Loss Component $=7 *$ Perdita operativa totale annua media +

$+7 *$ Perdita operativa totale annua media (per gli eventi con impatto superiore a $€ 10 \mathrm{mln}$.) +

$+5 *$ Perdita operativa totale annua media (per gli eventi con impatto superiore a $€ 100 \mathrm{mln}$.)

Si osserva che la formula proposta ha l'effetto di considerare due volte l'effetto economico degli eventi di perdita con impatto "intermedio" (compreso tra $€ 10 \mathrm{mln}$. e $€ 100 \mathrm{mln}$.), mentre l'effetto economico degli eventi di perdita estremi (con impatto superiore a $€ 100 \mathrm{mln}$.) viene considerato 2,9 volte ca; per tale via si genera quindi una sub-linearità della Loss Component rispetto all'effetto economico degli eventi di perdita, specificamente se estremi. In ogni caso, come evidenziato dal Comitato, per le banche non incluse nel bucket 1 la Loss Component è calcolata come una combinazione di importi delle perdite medie annue, costruita in modo da dare maggior peso relativo alle perdite di maggior importo.

A partire dalla Loss Component così determinata, si procede quindi a calcolare il moltiplicatore delle Internal Losses (o Internal Loss Multiplier, o ILM), attraverso la seguente formula:

$$
I L M=\text { Internal Loss Multiplier }=\operatorname{Ln}\left(\exp (1)-1+\frac{\text { Loss component }}{\text { BI component }}\right)
$$

dove il BI component è definito, coerentemente con la Tav. 2, come: 


$$
\text { BI component }= \begin{cases}0,11 * \mathrm{BI} & \text { se bucket } 1 \\ € 110 \mathrm{mln} .+0,15 *(\mathrm{BI}-€ 1 \mathrm{bln} .) & \text { se bucket } 2 \\ € 410 \mathrm{mln} .+0,19 *(\mathrm{BI}-€ 3 \mathrm{bln} .) & \text { se bucket } 3 \\ € 1,74 \mathrm{bln} .+0,23 *(\mathrm{BI}-€ 10 \mathrm{bln} .) & \text { se } \text { bucket } 4 \\ € 6,34 \mathrm{bln} .+0,29 *(\mathrm{BI}-€ 30 \mathrm{bln} .) & \text { se bucket } 5\end{cases}
$$

Si osserva, in particolare, che la funzione logaritmica utilizzata nel calcolo dell'ILM fa sì che esso cresca a tassi marginali tendenzialmente decrescenti al crescere della Loss Component; è opinione del Comitato che questa proprietà consenta di conseguire un ragionevole equilibrio tra la stabilità del requisito e la risk sensitivity del metodo SMA.

\section{Il calcolo del requisito di fondi propri con il metodo SMA}

Sulla base della proposta del Comitato, l'applicazione del metodo SMA richiederà alle banche di utilizzare, ai fini del calcolo del requisito di fondi propri per i rischi operativi, dieci anni di dati di perdite operative internamente registrate; ove una tale profondità storica non sia disponibile (ad esempio, per le banche che utilizzano attualmente il metodo BIA), sarà possibile in via transitoria utilizzare almeno 5 anni di dati di perdite operative interne, incrementando progressivamente la base dati interna fino al raggiungimento dell'intervallo temporale richiesto.

Ove neppure 5 anni di dati interni fossero disponibili, le banche dovranno calcolare il requisito di fondi propri SMA come se appartenessero al bucket 1, dunque (come mostrato dalla [9]) ponendolo pari alla BI Component.

La formula per il calcolo del requisito di fondi propri con il metodo SMA (o SMA Capital) è infatti la seguente:

$$
\text { SMA capital }=\left\{\begin{array}{lr}
\text { BI Component } & \text { se bucket } 1 \\
€ 110 \mathrm{mln} .+(\mathrm{BI} \text { Component }-€ 110 \mathrm{mln} .) \cdot I L M & \text { se bucket } 2-5
\end{array}\right.
$$

dove il moltiplicatore delle Internal Losses (o Internal Loss Multiplier, o ILM) è definito dalla [7].

Nella [9] si osserva che:

1. quando la Loss Component è esattamente pari alla BI component (ovvero la misura media storica, che tiene conto delle effettive perdite operative registrate storicamente dalla banca, è esattamente pari al livello medio delle perdite operative del bucket di riferimento), allora (sulla base della [7]) si ha che ILM = 1; conseguentemente, il requisito di fondi propri è esattamente pari alla BI component;

2. quando la Loss Component è inferiore alla BI component (ovvero la misura media storica, che tiene conto delle effettive perdite operative registrate storicamente dalla banca, è inferiore al livello medio delle perdite operative del bucket di riferimento), allora (sulla base della [7]) si ha che ILM $<1$; conseguentemente, il requisito di fondi propri è inferiore alla BI component;

3. quando la Loss Component è superiore alla BI component (ovvero la misura media storica, che tiene conto delle effettive perdite operative registrate storicamente dalla banca, è superiore al livello medio delle perdite operative del bucket di riferimento), allora (sulla base della [7]) si ha che ILM > 1; conseguentemente, il requisito di fondi propri è superiore alla BI component;

4. quando la Loss Component è pari a $\mathbf{0}$ (ovvero la banca non ha storicamente registrato alcuna perdita operativa negli ultimi 10 anni), allora (sulla base della [7]) ILM ha un limite inferiore al livello $\log [\exp (1)-1] \approx 0,541$; contestualmente, il requisito di fondi propri è circa pari all'11\% del BI.

Si rappresenta graficamente di seguito (cfr. Grafico 2) l'andamento dell'ILM (espresso come percentuale in ordinata) al variare della Loss Component, come definita dalla [6] in $€$ bln. (riportata in ascissa): 


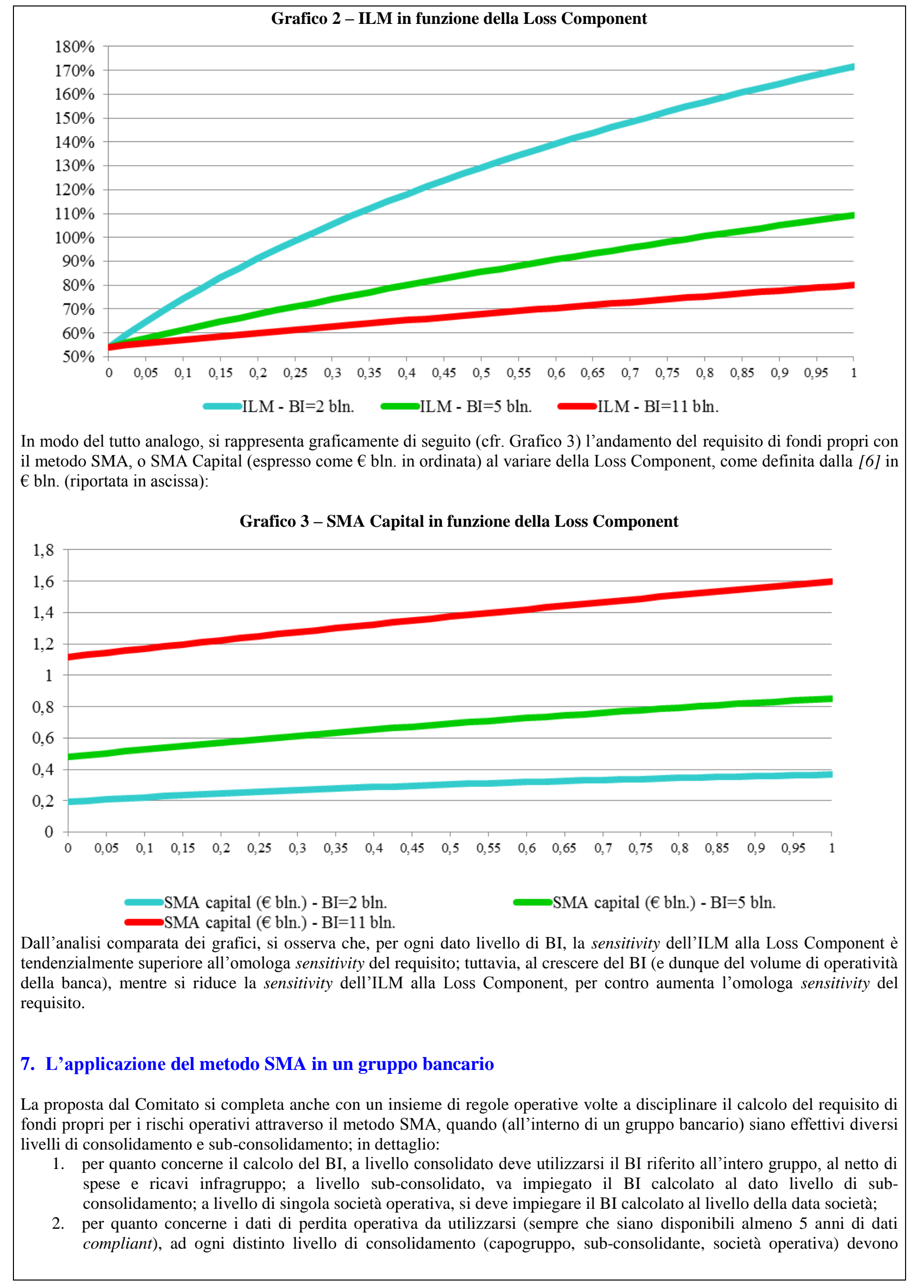


essere utilizzate solo le perdite operative riferite al perimetro contabile di consolidamento, senza includere eventi di perdita registrati da altre società del gruppo;

3. in ogni caso, sempre per quanto concerne i dati di perdita operativa da utilizzarsi, ad ogni distinto livello di consolidamento essi sono da utilizzarsi solo allorché il BI della legal entity di riferimento supera la soglia minima del bucket 2 ( $€ 1$ bln.);

4. infine, ad ogni distinto livello di consolidamento, qualora non siano disponibili almeno 5 anni di dati di perdita compliant, il requisito di fondi propri SMA dovrà essere calcolato come se la legal entity di riferimento appartenesse al bucket 1, dunque (come mostrato dalla [9]) ponendolo pari alla BI Component.

\section{Criteri di identificazione, raccolta e trattamento dei dati di perdita}

Venendo da ultimo ai requisiti di processo richiesti per l'applicazione del metodo SMA, il Comitato ritiene che un corretto processo di identificazione, raccolta e trattamento degli eventi di perdita interna è un prerequisito essenziale per il calcolo del requisito attraverso il metodo SMA; per garantire la coerenza nell'applicazione della Loss Component e per prevenire manipolazioni nel processo di loss data collection, si ritiene che le banche che utilizzano la Loss Component per il calcolo dello SMA Capital debbano soddisfare standard generali e specifici puntualmente elencati nel documento di consultazione.

In particolare, i dati di perdita da utilizzare nel metodo SMA dovranno essere compliant, ovvero rispettare una serie di stringenti requisiti per quanto concerne:

- la solidità del processo di raccolta delle perdite;

- l'integrità dei dati raccolti;

- la completezza dei dati raccolti;

- la storicizzazione dei dati raccolti;

- $\quad$ il trattamento dei dati raccolti.

Il Comitato distingue, nella sua proposta, standard (o criteri) generali e specifici per l'identificazione, la raccolta ed il trattamento dei dati di perdita; gli standard (o criteri) generali sono di seguito elencati:

- lunghezza temporale del periodo di osservazione dei dati di perdita operativa: il periodo di osservazione dei dati interni di perdita operativa deve essere di 10 anni; in sede di prima applicazione, è accettabile in via transitoria un periodo di almeno 5 anni se non sono disponibili dati compliant su un orizzonte temporale più lungo;

- documentazione dei dati di perdita operativa: i dati interni di perdita sono rilevanti se ricondotti chiaramente alle attività di business, ai processi tecnologici e alle procedure di risk management; pertanto devono esistere procedure e processi documentati per l'identificazione, la raccolta e il trattamento dei dati di perdita interna;

- raccordo delle perdite operative con gli event type : la banca deve essere in ogni caso capace di mappare i suoi dati interni di perdita storica sugli event type regolamentari, come stabiliti dall'art. 324 della CRR; questa attività di mapping deve avvenire secondo criteri documentati;

- Soglia minima di importo per gli eventi di perdita lorda: $i$ dati interni devono essere completi e rappresentare tutte le attività materiali della banca; una banca deve avere una appropriata soglia minima di perdita lorda per la raccolta dei dati interni di perdita: tale soglia può differire per event type, può differire da banca a banca e non può essere inferiore a $€ 10.000$ ( $€ 20.000$ in prima applicazione);

- informazioni minime sugli eventi di perdita: per ogni evento di perdita, una banca deve raccogliere: l'importo di perdita lorda, la data di riferimento (reference date), la data di accadimento (date of occurrence), la data in cui la banca ha scoperto l'evento (data di rilevazione, o date of discovery), la data in cui è avvenuto il primo passaggio a perdita o il primo accantonamento contabile (date of accounting), i recuperi avvenuti sulla perdita lorda, nonché informazioni descrittive sulle cause dell'evento di perdita (drivers). Il livello di dettaglio delle informazioni descrittive deve essere proporzionato all'ammontare delle perdita lorda;

- $\quad$ perdite multieffetto o pluriennali: una banca deve sviluppare criteri specifici per assegnare i dati di perdita originati da una funzione centralizzata (ex: IT department) o accaduti nel corso del tempo, se collegati;

- perdite di confine con il rischio di credito (credit boundary losses): le perdite operative collegate al rischio di credito (ex: perdite per non idonea formalizzazione delle garanzie), continuano ad essere considerate nella misurazione del requisito per il rischio di credito, e non contribuiscono al calcolo del requisito SMA;

- $\quad$ perdite di confine con il rischio di mercato (market boundary losses): le perdite operative collegate al rischio di mercato continuano ad essere considerate nella misurazione del requisito per il rischio operativo e contribuiscono al calcolo del requisito SMA.

Gli standard (o criteri) specifici, per l'identificazione, la raccolta ed il trattamento dei dati di perdita, sono di seguito elencati, distintamente per area tematica di riferimento:

1. relativamente alle perdite operative da utilizzare per il calcolo del requisito SMA, si richiede che:

a. (policy di gestione) la banca abbia una policy che specifica i criteri in base ai quali una perdita operativa registrata nel database delle perdite operative può essere inclusa nel dataset di calcolo del requisito SMA; 
b. (policy e procedure interne) la banca sviluppi normative e procedure interne per tutti gli aspetti rilevanti, incluse le informazioni minime sugli eventi di perdita, al fine di costruire un idoneo dataset di calcolo del requisito SMA a partire dai dati interni di perdita operativa;

2. relativamente alle definizioni di perdita lorda, perdita netta e recuperi, si richiede che la banca:

a. (definizioni) definisca: la perdita lorda come una perdita prima di ogni tipo di recupero; la perdita netta come la perdita lorda dopo aver considerato l'impatto dei recuperi; il recupero come un accadimento indipendente, riferibile all'evento di perdita originario, che avviene in un istante di tempo distinto da quello in cui è accaduto l'evento di perdita originario ed in cui la banca riceve (da una terza parte) denaro o benefici economici positivi;

b. (utilizzo) sia in grado di identificare, per ogni evento di perdita interna, l'ammontare di perdita lorda, $\mathrm{i}$ recuperi non assicurativi e i recuperi assicurativi; i recuperi assicurativi non possono essere portati in detrazione dell'importo di perdita utilizzato per calcolare il requisito di fondi propri;

3. relativamente al contenuto del database di calcolo del requisito SMA, si richiede che esso:

a. (contenuto obbligatorio) contenga obbligatoriamente:

i. aggravi e perdite dirette, inclusi i pagamenti effettuati, dovuti all'evento di perdita operativa;

ii. costi sostenuti in conseguenza dell'evento, incluse le spese esterne direttamente collegate all'evento (ex: spese legali) e le spese di riparazione/ripristino;

iii. svalutazioni (dirette o indirette) effettuate per far fronte agli effetti economici dell'evento;

iv. perdite collegate all'evento che non hanno ancora manifestato gli effetti a conto economico, in quanto appostate contabilmente in via provvisoria a c/vari (c.d. pending losses); le pending losses dovrebbero essere incluse nel dataset di calcolo del requisito SMA se di importo materiale, per dimensione e per tempo trascorso dall'appostazione;

v. impatti economici negativi contabilizzati in un esercizio, dovuti ad eventi di perdita operativa accaduti nell'esercizio precedente (c.d. timing losses); le timing losses materiali devono essere incluse nel dataset di calcolo del requisito SMA quando esse sono dovute ad eventi di perdita che producono effetti su più di un esercizio e comportano rischio legale;

b. (contenuto non ammesso) non contenga:

i. costi dei contratti di manutenzione di proprietà, impianti o equipaggiamenti;

ii. spese (interne od esterne) sostenute per rilanciare il business dopo l'accadimento di un evento di perdita operativa (ex: spese per migliorie, costi del risk assesment);

iii. premi assicurativi pagati per il trasferimento del rischio;

c. (orizzonte temporale di riferimento) contenga tutti gli eventi degli ultimi 10 anni; in particolare, il requisito SMA per ogni esercizio deve essere calcolato includendo le perdite lorde dell'esercizio, nonché gli aggiustamenti (di perdita lorda) registrati nell'esercizio su eventi con data di riferimento precedente di non oltre 10 anni la data di chiusura dell'esercizio;

d. (calcolo delle soglie) contenga tutti i dati necessari a calcolare le soglie rilevanti, considerato che (per calcolare gli eventi sopra le soglie di $€ 10$ bln. / $€ 100$ bln.) la perdita lorda dell'evento deve includere i sopra citati aggiustamenti di perdita lorda;

e. (data di riferimento dell'evento, o reference date) garantisca il rispetto dei criteri di determinazione della reference date di ogni evento; in dettaglio, la banca deve usare o la data di scoperta dell'evento (data di rilevazione, o date of discovery), o la data in cui è avvenuto il primo passaggio a perdita o il primo accantonamento contabile (date of accounting). Nessun'altra data è accettabile, neppure la data di accadimento; inoltre, per eventi di perdita riferiti ad eventi legali, la banca deve utilizzare una data non successiva alla date of accounting per inserire le perdite nel dataset di calcolo del requisito SMA; in tal caso, la date of accounting è la data in cui è stato fatto il primo accantonamento sulla base della perdita stimata;

f. (grouped losses) garantisca il rispetto dei criteri di determinazione delle grouped losses per ogni evento; in dettaglio, le perdite causate da uno stesso evento di perdita operativa, o da eventi collegati nel tempo, devono essere raggruppate e devono essere inserite (nel database di calcolo del requisito SMA) come una perdita operativa unica; inoltre, la policy di gestione (che specifica i criteri in base ai quali una perdita operativa registrata nel database delle perdite operative può essere inclusa nel dataset di calcolo del requisito SMA) deve formalizzare i criteri in base ai quali gli eventi di perdita devono essere raggruppati per il calcolo del requisito patrimoniale (quali circostanze, quali tipi di dati) e la metodologia da adottare; la banca deve anche documentare i margini di soggettività esercitabili nell'applicazione di tali criteri.

\section{Conclusioni}

La proposta del metodo SMA operata dal Comitato di Basilea evidenzia una complessiva revisione dell'ottica regolamentare, volta a mantenere l'adozione di processi avanzati di monitoraggio e gestione delle perdite operative, all'interno di un mutato framework di calcolo del requisito di fondi propri; per le banche italiane, le principali considerazioni sono state esposte 
dall'ABI all'interno del documento "Position Paper in response to BCBS consultation on the Standardised Measurement Approach for operational risk - June 2016", i cui elementi essenziali vengono di seguito brevemente riepilogati:

1. nonostante il Comitato ritenga che l'obiettivo del metodo SMA non sia quello di accrescere in modo significativo il requisito di fondi propri per il rischio operativo, le simulazioni operate su un campione di 18 banche italiane sui dati del triennio 2013-2015 hanno evidenziato che il passaggio dagli attuali metodi di misurazione al metodo SMA non risulta neutrale in termini di requisito di fondi propri: tutti gli intermediari appartenenti ai bucket di BI 2-5 registrerebbero un incremento di requisito, come pure oltre il 25\% degli intermediari appartenenti al bucket di BI 1; in particolare, per gli intermediari appartenenti ai bucket di BI 2-5, la mediana dell'incremento di requisito sarebbe del $46 \%$, ed un quarto di essi registrerebbe un incremento di requisito pari o superiore al $72 \%$;

2. il metodo SMA, come ogni metodo Standardizzato, si basa sull'assunzione implicita che esista un unico metodo di calcolo del requisito idoneo per tutte le giurisdizioni e tutte le linee di business (c.d. "one size fits all"), non risultando tuttavia sufficientemente valorizzate le differenze strutturali esistenti; in particolare, il Business Indicator utilizzato per il calcolo della BI Component all'interno del metodo SMA risulta scarsamente sensibile al rischio (cfr. supra, Grafico 3) ed è altamente correlato al Margine di Intermediazione Lordo, già adottato nell'esistente Metodo Standardizzato; inoltre, la formula proposta per il calcolo del Business Indicator BI prevede che i proventi/oneri straordinari, provenienti da cessioni di attività e/o da altri interventi organizzativi, comportino un aumento del requisito patrimoniale, determinando pertanto un costo implicito che dovrà essere preventivamente valutato in sede di definizione di eventuali operazioni omologhe a quelle citate;

3. dal punto di vista del framework di gestione delle perdite operative sviluppato dalle banche AMA (c.d. ORM framework), si osserva che esso risulta incorporato in modo significativo nel metodo SMA per quanto concerne gli strumenti di registrazione dei dati di perdita e di monitoraggio dei correlati eventi, mentre il requisito SMA risulta in generale meno sensibile agli esiti di efficaci ed affidabili politiche interne di gestione e riduzione delle perdite operative; tale riduzione di sensitivity comporta che (nel passaggio dai metodi AMA al metodo SMA) il processo di allocazione interna del capitale divenga meno efficiente, in quanto meno correlato alle perdite storiche e al grado di integrazione delle policy di risk management, che nelle banche AMA di norma tengono ora conto dei dati di perdita operativa anche all'interno dei processi ICAAP e di Stress Testing; inoltre, tale ridotta sensitivity implica anche, per le banche, minori incentivi impliciti ad investire in un ORM framework efficace (quanto a gestione delle perdite operative) e affidabile (nei termini di una identificazione prospettica delle future fonti di rischio operativo);

4. circa la dimensione della completezza dell'ORM framework, si osserva altresì che gli attuali processi di misurazione e gestione delle perdite operative prevedono, oltre all'utilizzo dei dati interni di perdita, anche l'uso di dati esterni, di analisi di scenario e di elementi che rispecchiano il contesto operativo e il sistema dei controlli interni; tutti questi ultimi elementi, invece, non trovano spazio entro il metodo SMA, nel quale i soli dati interni di perdita operativa sono considerati sufficienti a fornire una misura di rischio operativo risk sensitive $;{ }^{15}$ per tale via, la formulazione del mitigation plan in un contesto di adozione del metodo SMA potrebbe risultare non completa, in quanto la elaborazione delle azioni di mitigazione rischierebbe di concentrarsi sulle sole perdite operative interne, trascurando i fattori di contesto esterni e la valutazione del sistema dei controlli interni; un tale mitigation plan potrebbe quindi, in ultima analisi, distorcere la misurazione del rischio operativo prospettico a cui l'intermediario risulterebbe esposto; inoltre, il metodo SMA non contiene alcun incentivo a controllare il profilo di rischio operativo attraverso l'adozione di azioni di mitigazione, dato che esso non contiene alcuna componente forward looking, ed inoltre ciascuna perdita operativa (indipendentemente dall'adozione di azioni di mitigazione) genera un impatto diretto, in termini di requisito di fondi propri, per i successivi 10 anni;

5. con riferimento alla trasparenza e alla migliore comparabilità del requisito risultante dall'applicazione del metodo SMA, si osserva anche che (nel caso di sua applicazione a Gruppi Bancari caratterizzati da gradi intermedi di sub consolidamento) il coefficiente marginale applicato alla BI Component è in ogni caso calcolato a ogni singolo livello di consolidamento; ne consegue che un Gruppo composto da banche medio-piccole (aventi tutte BI inferiore a $€ 1$ bln.), ed in cui la Capogruppo supera invece la soglia prevista per il bucket 1, si trova ad avere un requisito consolidato che è superiore alla somma dei requisiti delle sue componenti, in quanto, a livello individuale, il BI di ogni singola banca non viene corretto per la loss component, ma a livello consolidato ciascuna singola banca si fa carico (in termini di requisito) delle perdite registrate dall'intero Gruppo; in altri termini, a livello individuale ma non a livello consolidato, a ciascuna singola banca viene applicato un moltiplicatore implicito della loss component (pari a 0) che è inferiore a quello applicato all'intero Gruppo. Tale approccio (che persegue una logica di calcolo del requisito di tipo top-down) risulta pertanto più penalizzante per le banche medio-piccole di bucket 1 che appartengono ad un Gruppo, rispetto alle banche di pari dimensioni che non fanno parte di un Gruppo;

6. data la (relativamente minore) risk sensitivity del metodo SMA rispetto ai metodi AMA, paradossalmente il passaggio dall'AMA allo SMA (pur generando misure più facilmente comparabili) tuttavia modificherebbe il level playing fields in direzione non coerente rispetto al rischio operativo effettivamente registrato, in quanto genererebbe un incremento tendenziale di requisito per le banche più virtuose e (specularmente) un effetto tendenzialmente

${ }^{15}$ Come si osserva all'interno del Position Paper dell'ABI, pag. 5, tale risk sensitivity appare perlatro non compiutamente adeguata: "The bank-specific loss data have been introduced in the formula in order to enhance the risk sensitivity of the previous standardized approach. However, we have observed that if calibrated in this way, the SMA does not achieve this goal because the weight of the business component is disproportionately higher than the 'risk-sensitive adjustment', frustrating the aim of having a capital absorption which is fairly fitted to bank's idiosyncratic risk profile." 
opposto per le banche che storicamente hanno registrato significativi volumi di perdite operative e/o eventi ad alto impatto economico;

7. inoltre, l'applicazione del metodo SMA determinerebbe una riduzione dell'incentivo all'impiego dei modelli interni per finalità di Pillar 2, di supporto al processo decisionale e di allocazione interna del capitale; ove infatti il requisito venisse calcolato con il metodo SMA, non vi è a priori alcuna garanzia che la conseguente allocazione del capitale regolamentare (che risulterebbe guidata più dalla contribuzione marginale al BI che non dall'incidenza relativa delle perdite storicamente registrate) possa risultare coerente con l'allocazione di capitale economico che potrebbe continuare a derivare dall'utilizzo dei modelli interni allo stato impiegati nei metodi AMA; il nuovo framework porrebbe quindi prospettive particolarmente sfidanti alle strutture di risk management, che dovrebbero efficacemente continuare a garantire l'allocazione del capitale economico utilizzando le metriche risk based interne, pur consapevoli che l'allocazione effettiva del capitale regolamentare seguirebbe metriche diverse; peraltro, la proposta del Comitato sul metodo SMA nulla prevede circa il ruolo del Pillar 2, ed ove il capital requirement di Pillar 2 dovesse continuare a trovare un floor in quello di Pillar 1, l'utilizzo dei modelli interni AMA per finalità di Pillar 2 diverrebbe di fatto inutile, con evidenti conseguenze anche in termini di processo SREP;

8. si osserva infine che il metodo SMA non riconosce nessun effetto (in termini di requisito di fondi propri) alla mitigazione del rischio conseguibile mediante il suo trasferimento per via assicurativa.

Successivamente al Position Paper dell'ABI, il Comitato di Basilea si è riunito tra il 28 e il 29 novembre 2016 a Santiago nel Cile, per valutare le complessive evoluzioni del framework di determinazione dei requisiti di fondi propri a fronte dei rischi di credito ed operativi; un'attenzione particolare è stata rivolta alla possibile reintroduzione di un approccio standardizzato per il rischio di credito, nonché all'introduzione dei metodi SMA per il rischio operativo. Il Comitato ha ribadito la marcata variabilità osservata negli attivi ponderati per il rischio (c.d RWA) tra le istituzioni bancarie delle diverse giurisdizioni, e per conseguenza la ridotta comparabilità degli stessi in relazione alle diverse tipologie di rischio. Al fine di ridurre detta variabilità, il Comitato intende ricorrere (nel c.d. framework Basilea 4) ad un più ampio impiego di approcci di tipo c.d. Standardizzato, unitamente all'applicazione di soglie minime (floor). Con specifico riferimento al calcolo del requisito di fondi propri per i rischi operativi, il metodo SMA di nuova proposizione dovrebbe comportare che le banche di maggiori dimensioni subiscano un maggior impatto rispetto a quelle di minore dimensione, poiché l'esposizione ai rischi operativi dovrebbe crescere più che proporzionalmente con l'incremento del BI, che rappresenta un'approssimazione delle dimensioni della banca. In ogni caso, si osserva che l'approccio proposto comporta un "costo fisso" in termini di RWA (anche in assenza di perdite operative interne che valorizzino la Loss Component), proporzionale alla dimensione aziendale, che nella logica risulta estremamente simile alle implicazioni della proposta di floor per i metodi AIRB per il rischio di credito. Tale "costo fisso" prescinde, nelle intenzioni del Comitato, dal livello di efficienza del sistema di gestione dei costi operativi, il quale può solo intervenire a mitigare l'incremento del requisito complessivo al di sopra di tale "costo fisso"; pertanto, se con gli attuali metodi AMA una maggiore efficienza del sistema di gestione dei rischi operativi si traduce in una minore allocazione del capitale, diversamente (con il nuovo metodo SMA), detta efficienza comporta solo un minor incremento del requisito stesso.

Per le ragioni citate, nonché per i conseguenti incrementi patrimoniali che le proposte del Comitato potrebbero generare, lo stesso Parlamento europeo ha evidenziato che dette proposte non saranno ragionevolmente incorporate nella direttiva sui requisiti patrimoniali (c.d. CRD 5) attualmente in formulazione, poiché il framework Basilea 4 introdurrebbe un sistema di fatto impermeabile al contesto soggettivo e di mercato, che renderebbe inutilizzabili i costosi modelli di cui si sono dovute dotare le banche, grandi e medio-grandi, negli ultimi anni.

In tal senso, il proposto framework di calcolo del requisito di fondi propri, che accresce la comparabilità delle risultanze, in ogni caso riduce la risk sensitivity delle metriche utilizzate; le più evidenti conseguenze di tale evoluzione si registrerebbero principalmente con riferimento a quei processi che attualmente utilizzano le attuali metriche per finalità gestionali, al fine di raccordare più agevolmente le misure di capitale regolamentare ed economico: in tale ambito, infatti, l'evoluzione del contesto regolamentare finora analizzata determinerebbe il venir meno delle opportunità che, allo stato attuale, incentivano l'utilizzo dei modelli interni per finalità gestionali, cosicché l'obiettivo proposto dal Comitato (realizzare una più agevole comparabilità del requisito di fondi propri per i rischi operativi tra le diverse istituzioni finanziarie) potrebbe essere conseguito al costo di un incremento di rischio sistemico, conseguente all'adozione di tecniche meno risk sensitive anche per finalità gestionali.

Risulta infine indubbio che il metodo SMA, riducendo la risk sensitivity del requisito di fondi propri, non introduce incentivi per le banche ad adottare azioni di mitigazione tempestive a fronte dei rischi operativi di recente manifestazione, quali quelli legati alla digitalizzazione dell'attività bancaria: in prospettiva, l'adozione del metodo SMA, potrebbe comportare minore tempestività nella mitigazione di tutti quei rischi che si manifestano inizialmente con impatti di bassa entità, i quali tuttavia possono preludere a perdite operative di maggiore magnitudine se non tempestivamente affrontati tramite l'adozione di idonei piani di mitigazione.

Giacomo Petrini e Camillo Giliberto

\section{Bibliografia}

- REGOLAMENTO (UE) N. 575/2013 DEL PARLAMENTO EUROPEO E DEL CONSIGLIO;

- Consultative Document Standardised Measurement Approach for operational risk March 2016 


\title{
I paradigmi di apprendimento non supervisionato per reti neurali in campo finanziario: progettazione di self-organizing maps per il rintracciamento di anomalie di mercato
}

\author{
di Pier Giuseppe Giribone (Banca Carige) - Alessia Cafferata (Università di Genova )
}

\section{ABSTRACT}

The algorithms based on artificial intelligence together with the self-computing numerical methodologies inspired by natural processes are gaining importance in financial issues.

The aim of this article is to implement an automatic method for organizing and clustering the information observed on secondary markets, focusing the attention on the recognition of potential anomalies.

The methodology, used to highlight potential opportunities of trading, is constituted by a non-supervised neural network, designed to clustering the data, known as Kohonen Network or Self-Organizing Map (SOM).

The article is divided into three parts:

The first one shows how the methodology works, that is how the structure of the network is defined, how the training procedure is projected and which algorithm must be implemented for a correct mapping of the experimental data.

In the second part, we proceed to write and validate the code in a development environment oriented to mathematical modelling; in particular Matlab language has been utilized.

In the last part, examples of the applications of SOM in the recognition of anomalies in bond markets are provided.

\section{Key Words:}

Unsupervised learning network, Self-organizing maps (SOM), Kohonen Networks, Artificial Neural Networks (ANN), clustering algorithm, data mining

\section{Introduzione}

Gli algoritmi basati sull'intelligenza artificiale, sulle strategie di autoapprendimento e le metodologie numeriche di softcomputing ispirate ai processi naturali sono di interesse crescente nel campo finanziario: essi consentono infatti di analizzare più rapidamente una notevole quantità di dati finanziari. Il fine del presente articolo è quello di implementare una metodologia automatica di organizzazione e classificazione delle informazioni registrate sui mercati secondari mobiliari atta all'individuazione di potenziali anomalie. Un problema di forte rilevanza nell'ambito del trading, infatti, è quello di determinare le potenziali opportunità di arbitraggio tra gli strumenti finanziari che vengono scambiati nei mercati: numerosi contributori forniscono differenti quotazioni associate ad un singolo prodotto, rimandando all'abilità dell'operatore l'analisi di convenienza del livello dei prezzi tenendo anche in considerazione i volumi a questi associati. La metodologia da noi proposta può essere considerata un buono strumento da essere impiegato a tal scopo, ovvero finalizzato ad evidenziare interessanti anomalie di prezzo, e, in ultima analisi, potenziali opportunità di trading. L'algoritmo di calcolo impiegato per il rintracciamento di queste possibili opportunità di trading è costituito da una rete neurale non supervisionata, adibita al clustering di dati, nota in letteratura come Kohonen Network o Self-Organizing Map (SOM). Tale tipologia di rete ha il vantaggio di non richiedere a-priori di specificare il numero di partizioni da implementare, differentemente da altre metodologie di clustering, come il K-means e il Fuzzy C-means.

L'articolo si suddivide in tre parti:

La prima presenta il funzionamento della metodologia, ovvero come viene definita l'architettura di rete, come si progetta la procedura di addestramento e quale algoritmo deve essere implementato per una corretta mappatura dei dati sperimentali.

Nella seconda si procede alla scrittura e relativa validazione del codice in un ambiente di sviluppo orientato alla modellizzazione matematica, nel particolare si è impiegato il linguaggio per l'elaborazione numerica Matlab ${ }^{\circledR}$.

Nell'ultima parte si forniscono degli esempi di applicazione delle SOM in ambito finanziario, che consentono l'individuazione di anomalie nel mercato obbligazionario.

\section{Il funzionamento di una Self-Organizing Map}

Negli anni Ottanta, Teuvo Kohonen, studiando il funzionamento biologico di come le reti neurali organizzano e classificano le informazioni acquisite, sviluppò le self-organizing feature maps, meglio note in letteratura come self-organizing maps o SOM [1], [2], [3], da cui discende un paradigma di rete neurale (ANN - Artificial Neural Network) caratterizzato dalla proprietà che l'output è lo stato stesso della rete, ovvero come questa si organizza, apprendendo completamente in modo non supervisionato.

A differenza del funzionamento delle altre ANN, ad esempio le feed-forward neural network adatte per il fitting [4] e l'estrapolazione [5], per le SOM non è necessario conoscere quale valore è calcolato dal neurone, ma solamente sapere quale neurone interviene attivamente nella rete. 
L'analogia biologica è decisamente verosimile: in natura i neuroni sono connessi con certi muscoli, il primo problema gestito dal nostro centro nervoso non è conoscere quanto un muscolo sia contratto, ma quale sia il muscolo attivato. In altre parole: non si è subito interessati all'output esatto fornito dal neurone, ma nel riconoscere il neurone che fornisce l'output richiesto. I neuro-scienziati considerano di gran lunga il principio di funzionamento delle SOM molto più collegato alla biologia rispetto ad altri paradigmi ispirati al funzionamento di come il cervello umano elabora i dati acquisiti dai sensi.

\subsection{L'architettura di una SOM}

Tipicamente una SOM ha il compito di organizzare un input complesso e di elevate dimensioni ( $N$ ) in aree spaziali, partizionate da celle ( grids), di dimensioni meno elevate rispetto a quelle di partenza ( $G$ dimensionali).

Questo processo (mapping) permette di generare una mappa rappresentativa del contesto studiato, potendo evidenziarne le caratteristiche salienti e riducendone la sua complessità $(G<<N)$.

Al fine di generare questa mappa, la SOM inizialmente alloca secondo una griglia di partenza ( $G$ - dimensionale) i punti all'interno dello spazio vettoriale complesso di riferimento ( $N$ - dimensionale).

Durante l'algoritmo di apprendimento, i punti costituenti la SOM modificheranno la propria posizione nello spazio e, conseguentemente, la topologia della rete, in modo da ricoprire al meglio le posizioni assunte dai punti di partenza percepite dai suoi neuroni. Proseguendo con l'analogia naturale, significa che ciascun neurone può essere attivato per interpretare una determinata posizione nello spazio di ingresso (ambiente esterno): maggiori stimoli provengono da un'area e maggiore sarà il numero di neuroni attivati per codificare il segnale percepito.

Tale logica consente al cervello di isolare le zone "interessanti", in cui si addenseranno i punti della griglia (neuroni assegnati alla codifica dell'informazione) da quelle marginali, nelle quali saranno assegnati pochi ricettori.

Riassumendo i concetti sinora esposti, si può affermare che vengono definiti due spazi distinti in cui le SOM operano:

- lo spazio di input $N$ - dimensionale

- la griglia $G$ - dimensionale sulla quale vengono disposti i neuroni che interpretano le relazioni di vicinanza in funzione dei segnali ricevuti dall'ambiente, ovvero dalla disposizione spaziale dei punti presenti nello spazio di input. Modificando le neighborhood relationships viene determinata la topologia stessa della rete (network topology).

In una griglia monodimensionale, i neuroni di partenza sono generalmente disposti in modo equi-distanziato su un segmento. Una griglia bidimensionale può essere progettata generalmente secondo tre topologie:

- rettangolare (le connessioni tra neuroni contigui formano angoli retti costituendo una griglia rettangolare regolare)

- esagonale (le connessioni tra neuroni contigui formano angoli di sessanta gradi costituendo una griglia di triangoli equilateri) - casuale (i punti della rete sono generati casualmente all'interno dello spazio vettoriale di input)

Le più diffuse griglie sono le prime due in quanto la configurazione iniziale risulta più ordinata per costruzione iniziale. La regolarità della partizione è rappresentata nella Figura 1: a sinistra si è progettata una griglia rettangolare (detta anche matriciale), a destra una esagonale (detta anche "ad alveare").
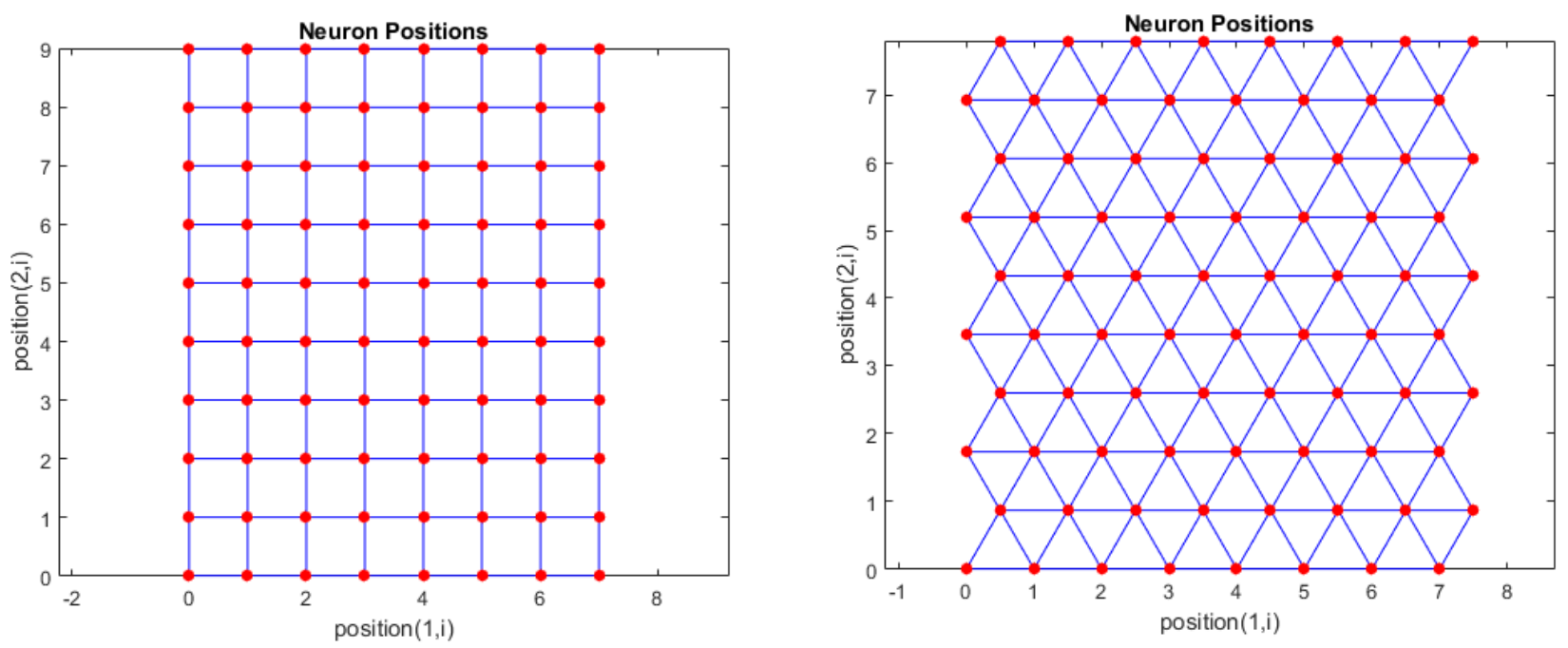

Figura 1. Le Network topology più diffuse per la progettazione di una SOM

Topologie a più dimensioni possono essere trattate dalle SOM, ma non vengono nella pratica molto impiegate perché la gestione tra le relazioni di vicinanza sono complesse da visualizzare e da interpretare in modo intuitivo. 
Similmente ai neuroni di una RBF (Radial Basis Function) network, l'unità base di calcolo di una Kohonen network (SOM neuron), $k$, non occupa una posizione fissa, $c_{k}$ nello spazio di input.

Caratteristica comune alle reti neurali e pertanto anche delle SOM [6], [7], è il processo di addestramento dei suoi neuroni, che si riconduce, nella sua essenza, ai seguenti step:

Input: un valore arbitrario $p$ dello spazio degli input $\mathfrak{R}^{N}$;

Calcolo della distanza euclidea tra ogni neurone $k$ e $p$, ossia il calcolo della norma: $\left\|p-c_{k}\right\|$

Attivazione di un neurone: il centro di calcolo con la distanza più ravvicinata all'input, denominato $i$, diventa attivo, mentre tutti gli altri rimangono inattivi. Questo modo di procedere è denominato "winner-takes-all scheme".

Output: la procedura evidenzia quale neurone è diventato attivo.

\subsection{L'algoritmo di training}

Il training di una SOM permette alla mappa di esplorare correttamente il dominio e pertanto è considerato il cuore della tecnica [8]. Data l'importanza che riveste, è bene approfondire più nel dettaglio gli step procedurali.

\section{Inizializzazione}

La rete distribuisce casualmente i centri $c_{k} \in \mathfrak{R}^{N}$ nello spazio vettoriale di riferimento.

Creazione di un pattern

Uno stimolo (stimulus), ossia un punto $p$, è selezionato dallo spazio vettoriale degli input $\mathfrak{R}^{N}$.

Misurazione della distanza

Per ogni neurone $k$ della rete viene stimata la distanza euclidea: $\left\|p-c_{k}\right\|$.

"Winner-takes-all” scheme

Si determina il neurone vincente $i$, ossia quello con la distanza minore da $p$.

Questo deve pertanto soddisfare la seguente condizione: $\left\|p-c_{i}\right\| \leq\left\|p-c_{k}\right\|, \forall k \neq i$

Adattamento dei centri

I centri $c_{k}$ sono mossi nello spazio vettoriale degli input secondo la learning rule:

$\Delta c_{k}=\eta(t) \cdot h(i, k, t) \cdot\left(p-c_{k}\right)$

$c_{k}(t+1)=c_{k}(t)+\Delta c_{k}(t)$

Dove la variazione $\Delta c_{k}$ è semplicemente aggiunta alla posizione corrente ricoperta dai centri $c_{k}$.

L'ultimo fattore mostra che il cambio nella posizione dei neuroni $k$ è proporzionale sia alla distanza di $p$ sia al coefficiente di apprendimento tempo-variante $\eta(t)$.

La topologia di rete esercita la sua influenza mediante la funzione $h(i, k, t)$, detta topology function : essa è definita sulla griglia, deve essere unimodale, ossia deve presentare un solo massimo, il quale è prossimo al neurone vincente $i$.

Una delle scelte più comuni è quella di adottare per $h$ una campana gaussiana: infatti essa è unimodale e con un massimo vicino a $0[8]$.

In aggiunta a ciò, la sua ampiezza può essere modificata dal parametro $\sigma$, che può essere utilizzato per ridurre la distanza al passare del tempo: è sufficiente mettere in relazione la dipendenza temporale con $\sigma$, ottenendo come risultato una funzione monotonamente decrescente, $\sigma(t)$.

La funzione topologica dotata delle caratteristiche appena descritte ed impiegata più comunemente in letteratura è definita: 
$h(i, k, t)=\exp \left(-\frac{\left\|g_{i}-c_{k}\right\|^{2}}{2 \sigma(t)^{2}}\right)$

dove $\left\|g_{i}-c_{k}\right\|^{2}$ indica la distanza tra il centro $c_{k}$ e l'i-esimo punto appartenente alla griglia.

Il tasso di apprendimento è una funzione monotona decrescente: questo generalmente assume valori dipendenti dalla topologia di rete che generalmente ricadono nell'intervallo: $0.01 \leq \eta \leq 0.6$ [8].

E' una condizione necessaria al fine di un corretto addestramento delle reti di Kohonen la verifica della diseguaglianza: $h \cdot \eta \leq 1[8],[1]$.

\section{Esempi di funzionamento dell'algoritmo su dati simulati}

La tecnica di clustering dati mediante SOM è stata scritta usando il software di elaborazione numerica Matlab®. Prima di impiegare la metodologia in campo finanziario, si rende necessario applicare un criterio di validazione del codice, al fine di essere confidenti di un corretto funzionamento della libreria sviluppata.

A tal fine si sono strutturati due test, normalmente impiegati per verificare il corretto adattamento della rete neurale artificiale ai dati sperimentali [10].

\subsection{Adattamento della rete su un campione di punti sparsi nel dominio secondo una distribuzione uniforme}

Vengono generati casualmente 5000 punti nel dominio $[0 ; 1]^{2}$ e, partendo da una SOM caratterizzata da una griglia di partenza avente 100 nodi anch'essi distribuiti casualmente (Random Network Topology) in un intervallo più grande $[-0.5 ; 1.5]^{2}$, si verifica la corretta mappatura della rete sui dati sperimentali. (Figura 2) [10]

Il risultato atteso è che, iterazione dopo iterazione, la griglia di partenza ricopra progressivamente più punti possibili nella porzione spaziale dove sono stati collocati i punti di prova, i quali, essendo stati generati secondo una distribuzione uniforme, la mappa non deve riconoscere particolari pattern. (Figura 3)

In altre parole, il valore atteso è che la SOM collocando i suoi neuroni nel quadrato unitario lo ricopra uniformemente nei vincoli spaziali.

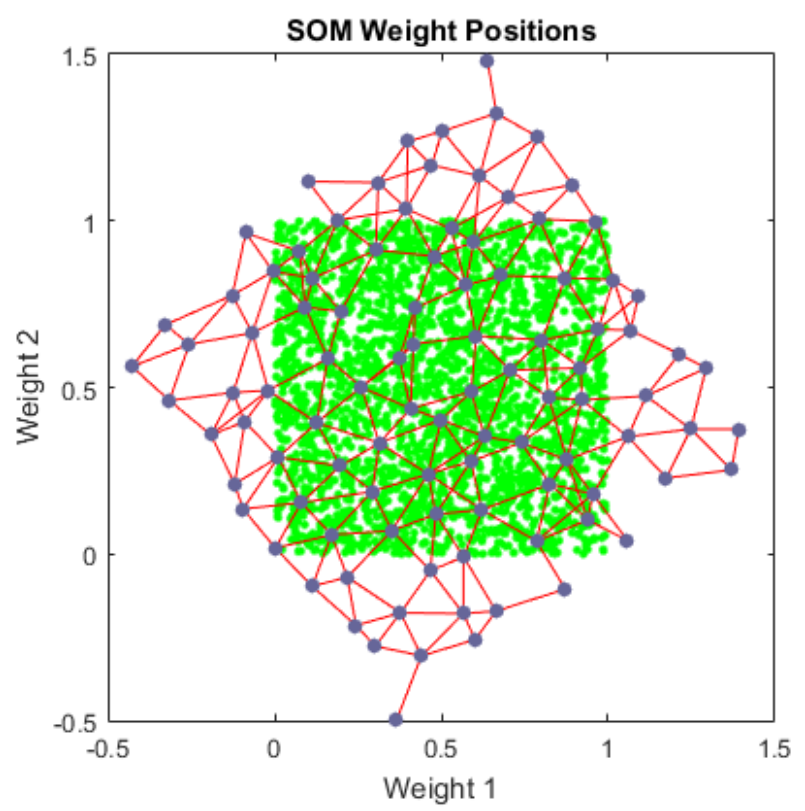

Figura 2. Disposizione iniziale dei punti e dei nodi della rete per il test presentato nel paragrafo 3.1 

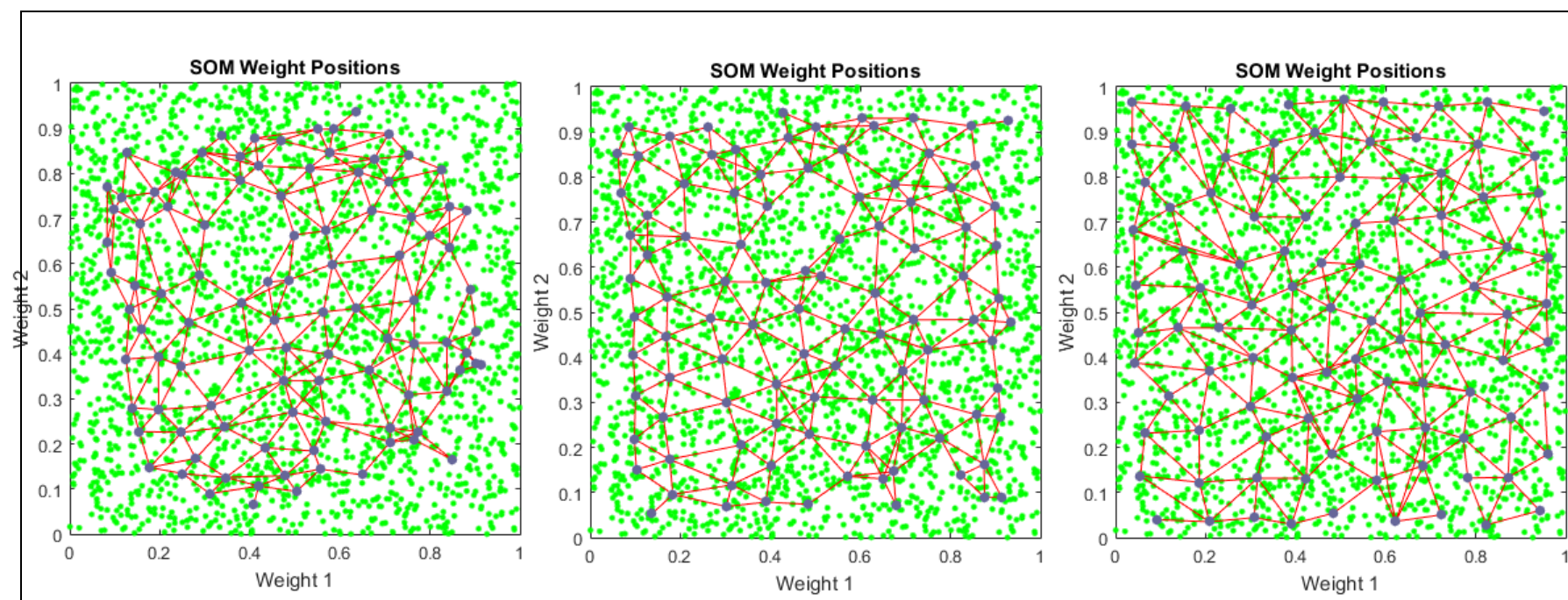

Figura 3. Apprendimento della SOM rispettivamente dopo 10, 100 e 1000 iterazioni della routine principale (epoche)

Le illustrazioni testimoniano il corretto apprendimento della rete neurale non-supervisionata e, pertanto, l'esatta disposizione dei suoi neuroni all'interno del dominio sperimentale.

\subsection{Adattamento della rete su un campione di punti sparsi nel dominio secondo una distribuzione gaussiana}

Vengono generati casualmente 5000 punti sparsi nel dominio secondo una distribuzione normale standard, ovvero una gaussiana di media zero e scarto quadratico medio unitario $(\sigma)$. La SOM utilizzata per questo test presenta una griglia di partenza "ad alveare" (hex-grid topology) formata da 72 nodi. [10]
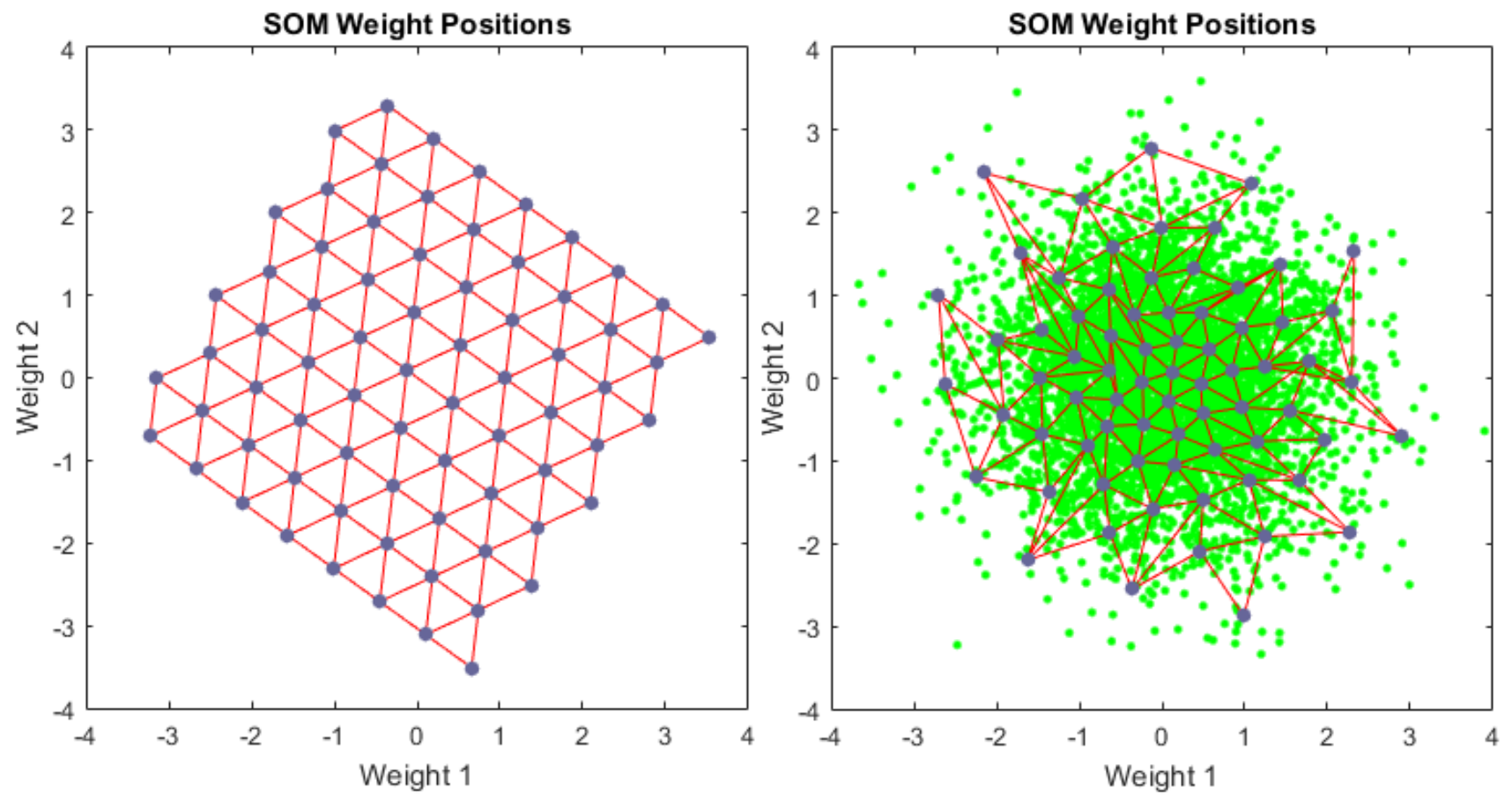

Figura 4. Disposizione iniziale e finale dei neuroni costituenti la griglia della SOM (Training di 5000 epoche)

Dopo 5.000 epoche la SOM ha mappato correttamente il dominio sperimentale concentrando la sua attenzione in una circonferenza di centro $(0 ; 0)$ e raggio pari a circa 3 volte la deviazione standard (Figura 4). Si può pertanto concludere che l'algoritmo è stato in grado di isolare gli outliers, identificati come quei punti disposti fuori dal cerchio con centro nell'origine e raggio superiore ai $3 \sigma$.

\section{Applicazione della metodologia di soft-computing in campo finanziario}

Effettuata la validazione teorica-statistica del modello, si procede ad applicare le SOM in qualità di strumento per l'individuazione automatica di potenziali anomalie di mercato. In questo contesto, si applica la metodologia presentata nel 
mercato secondario dei titoli obbligazionari: ogni potenziale transazione è caratterizzata da una quantità che si vuole comprare o vendere e da un prezzo al quale il trader desidererebbe concludere lo scambio.

Isolando su un piano queste due variabili fondamentali, si riesce, mediante l'ausilio di una rete neurale non-supervisionata, a partizionare lo spazio in regioni che consentono di individuare e classificare le zone in cui si osserva una maggiore concentrazione di punti da quelle in cui questi sono più dispersi.

La metodologia sarebbe quindi in grado di discriminare le anomalie di mercato (intese come prezzi e/o volumi più alti o bassi rispetto a quelli normalmente registrati) trattandoli come outliers nel piano di riferimento: pertanto tutti i punti non compresi all'interno della rete costituiscono potenziali anomalie finanziarie, che la procedura automatica è in grado di segnalare all'operatore.

Si procede, quindi, a descrivere la metodologia in applicazione alle quotazioni registrate sul mercato nel primo pomeriggio di martedì 19 luglio 2016 su due titoli obbligazionari quotati da contributori: il primo caso è un bond con cedola $13 / 8$ e scadenza 2026, il secondo un titolo con cedola 2 7/8 e scadenza 2020.

Si precisa che la scelta del numero dei nodi della mappa $(G)$ è un parametro di ingresso dell'algoritmo, mentre le osservazioni di prezzo/volume $(N)$ sono in funzione delle contribuzioni del titolo nel tempo di osservazione. Maggiore è il numero dei nodi, minore è la capacità della rete di concentrarsi nelle aree di maggiore concentrazione (infatti se $N=G$ i nodi si allocherebbero esattamente sopra i punti rappresentanti le osservazioni di mercato in quell'istante).

D'altro canto una mappa dotata di un numero di neuroni estremamente basso potrebbe fornire un numero troppo elevato di warning. Si considera, pertanto, un buon trade-off operativo porre $G=N / l$ con $l \in[3,5]$.

Si preferisce impiegare una topologia iniziale di rete deterministica al fine di mantenere una tracciabilità e riproducibilità dei passaggi che hanno portato ad evidenziare le potenziali anomalie: in particolare, negli esperimenti effettuati viene usata una mappa esagonale.

Per terminare la giustificazione dei parametri impiegati nella progettazione della SOM, si riporta che si è scelto un training di 1.000 epoche: poiché si è verificato sperimentalmente essere un buon compresso tra affidabilità dei risultati evidenziati e tempo computazionale impiegato dalla mappa di Kohonen per riaggiornarsi in un contesto finanziario in cui la variazione delle contribuzioni è prossima al real-time.

\subsection{Individuazione delle anomalie di prezzo/volume per il primo bond}

Partendo da una topologia esagonale della rete composta da 9 nodi, si procede all'addestramento non-supervisionato della stessa con l'obiettivo di individuare nel piano di riferimento (volumi/prezzi) i potenziali outlier. I dati sono stati scaricati mediante la funzione “All Quotes” (ALLQ) dell'info-provider Bloomberg®. La Figura 5 mostra la SOM prima (immagine di sinistra) e dopo il training di 1000 epoche (immagine di destra) nel piano cartesiano [quantità (espressa in milioni); prezzo (espresso in percentuale)]. I punti blu sono i neuroni della ANN ( $G=9$ ), i punti in verde sono i dati sperimentali osservati sul mercato ( $N=36$ ), le linee rosse determinano le partizioni spaziali che caratterizzano la rete. Sono considerati outliers, e pertanto potenziali anomalie di mercato, i punti non compresi all'interno della mappa. Si può osservare come la libreria sia riuscita ad evidenziare come, ad esempio a parità di quantità (1 milione), il titolo ha registrato proposte di scambio con prezzi significativamente diversi rispetto alla media (106,4\% e quotazioni superiori a $107,7 \%)$. La rete ha correttamente escluso i prezzi aventi un volume pari ai 2 milioni, in quanto il baricentro del sistema è sicuramente compreso tra mezzo milione e un milione.
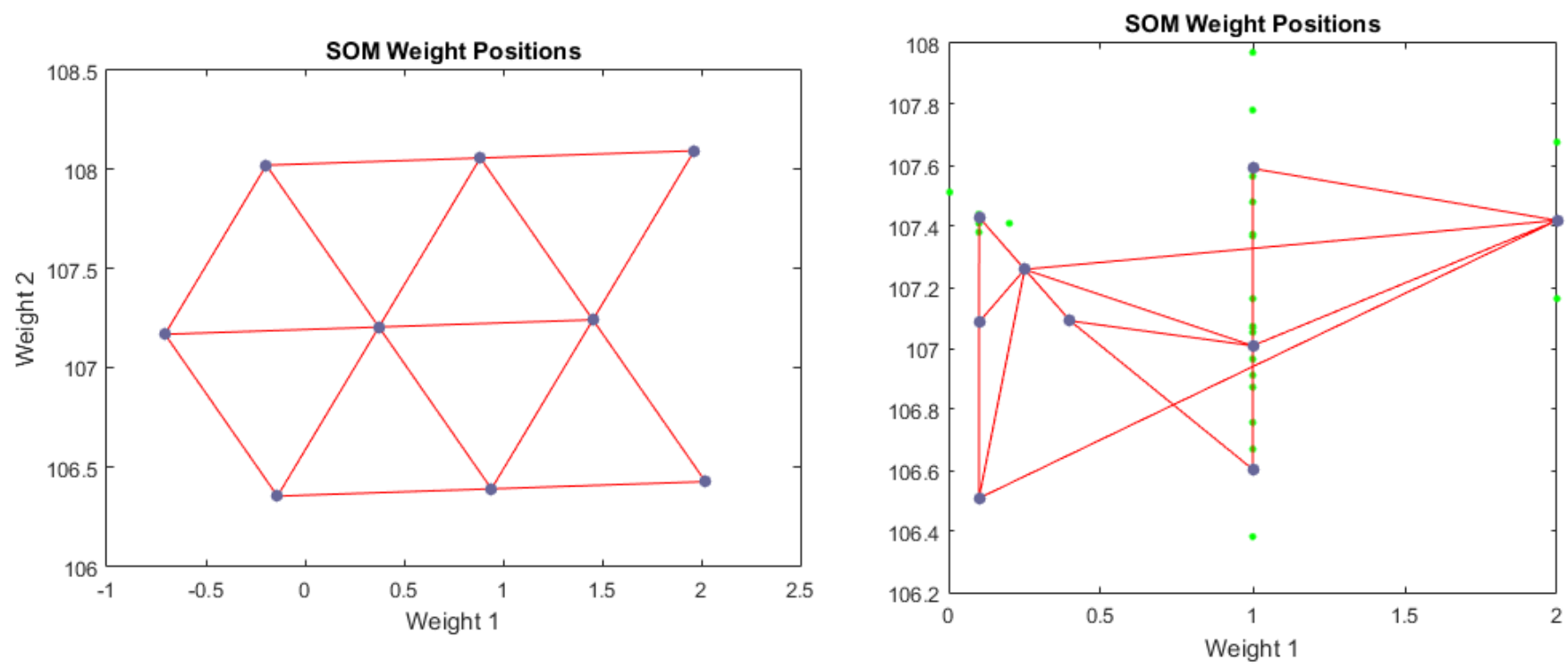

Figura 5. Individuazione delle anomalie di mercato per il primo titolo in esame 


\subsection{Individuazione delle anomalie di prezzo/volume per il secondo bond}

Analogamente a quanto fatto nel precedente paragrafo, si procede alla progettazione di una SOM caratterizzata da una topologia esagonale con un numero di neuroni pari a $G=9$ per un numero complessivo di dati sperimentali analizzati pari a $N=42$

La Figura 6 evidenzia la corretta clusterizzazione dello spazio di riferimento avvenuta con un addestramento della rete durato 1000 epoche. Le convenzioni grafiche e le unità di misura descrittive delle grandezze finanziarie impiegate sono uguali a quelle utilizzate in precedenza.

La partizione spaziale generata dalla SOM è stata in grado di individuare i potenziali outliers: ovvero le due proposte di scambio con volume superiore al milione ed i deal con una quotazione superiore al $109.9 \%$ e inferiore ai $108.4 \%$ in corrispondenza di una quantità inferiore o pari al mezzo milione.
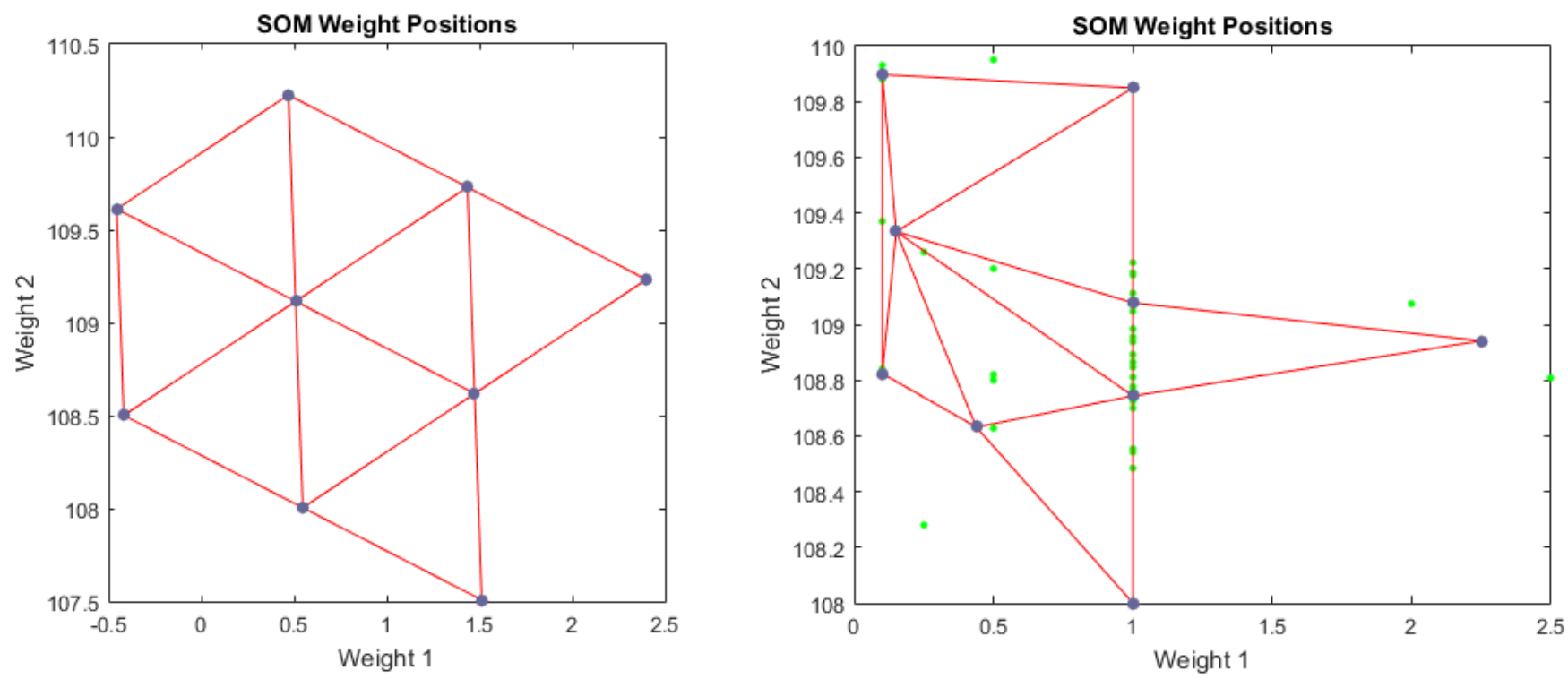

Figura 6. Individuazione delle anomalie di mercato per il secondo titolo in esame

\section{Conclusioni}

L'articolo ha evidenziato come le Self-Organizing Maps possano essere impiegate per progettare procedure automatiche di individuazione di anomalie di mercato e, conseguentemente, potenziali opportunità di trading. L'algoritmo impiegato si è dimostrato essere una metodologia efficiente sia dal punto di vista teorico che nella sua applicazione pratica nel mercato obbligazionario.

Le reti di Kohonen non dovendo, a differenza di molti altri algoritmi di clustering, quali, ad esempio il K-means e la Fuzzy Cmeans [9], specificare aprioristicamente il numero di suddivisioni da effettuare si sono rivelate uno strumento flessibile di organizzazione delle informazioni finanziarie.

Si reputa interessante per il prosieguo dello studio applicare la metodologia presentata ad altri ambiti delle scienze finanziarie dove è di fondamentale importanza la corretta classificazione delle informazioni (finanza sperimentale, comportamentale $\mathrm{e}$ sistemi di algo-trading).

Un'ulteriore estensione del presente lavoro potrebbe essere costituita dall'introduzione di mappe di calore (heating maps) che consentono di analizzare e descrivere con ulteriori importanti dettagli le informazioni dei mercati finanziari.

Infine, potrebbe essere oggetto di un ulteriore approfondimento integrare l'output fornito dalle SOM con altri algoritmi di clustering che consentono di organizzare i dati senza la necessità di specificare a-priori il numero di partizioni da effettuare, tra questi si cita il t-SNE (t-Distributed Stochastic Neighbor Embedding) e le metodologie di Kernel.

Pier Giuseppe Giribone e Alessia Cafferata

\section{Bibliografia}

[1] T. Kohonen, "Self-organized formation of topologically correct feature maps", Journal of Biological Cybernetics, 43:5969, 1982.

[2] T. Kohonen, "Self-Organization and Associative Memory", Springer-Verlag, Berlin, third edition, 1989. 
[3] T. Kohonen, "The self-organizing map", Journal of Neurocomputing, 21 (1-3):1-6, 1998.

[4] P. G. Giribone, S. Ligato, O. Caligaris - "Applicazioni delle reti neurali feed-forward per la ricostruzione di superfici di volatilità" - AIFIRM Magazine, Associazione Italiana Financial Industry Risk Managers, 2015

[5] P. G. Giribone, O. Caligaris - "Modellizzare la curva dei rendimenti mediante metodologie di apprendimento artificiale: analisi e confronto prestazionale tra le tecniche regressive tradizionali e le reti neurali" - AIFIRM Magazine, Associazione Italiana Financial Industry Risk Managers, 2015

[6] P. G. Giribone - "Teoria ed applicazioni delle reti neurali artificiali feed-forward nella Response Surface Methodology" Seminario tenuto al Primo anno Laurea Magistrale Ingegneria Gestionale dell'Università di Genova

[7] O. Caligaris - "Le reti neurali" - Lettera matematica PRISTEM, 61:20-28, 2007

[8] D. Kriesel - "A brief introduction to Neural Network" - Author Website, 2005

[9] P. G. Giribone, O. Caligaris, S. Fioribello - "L'algoritmo della Fuzzy C-Means clustering come tecnica automatica per l'individuazione di anomalie di mercato" - AIFIRM Magazine, Associazione Italiana Financial Industry Risk Managers, 2016 [10] M. H. Beale, M. T. Hagan, H. B. Demuth - "Neural Network Toolbox, User’s Guide" - The MathWorks, Inc., 2014 
Risk Management Magazine

Anno 12, n 2 Maggio - Agosto 2017

Direttore Responsabile

Maurizio Vallino

Condirettore

Corrado Meglio

Consiglio Scientifico

Giampaolo Gabbi (Direttore del Consiglio Scientifico),

Paola Bongini,

Anna Bottasso,

Ruggero Bertelli,

Ottavio Caligaris,

Simona Cosma,

Paola Ferretti,

Andrea Giacomelli,

Adele Grassi,

Pier Giuseppe Giribone,

Duccio Martelli,

Anna Grazia Quaranta,

Pasqualina Porretta

Enzo Scannella,

Cristiana Schena,

Giuseppe Torluccio

Comitato di redazione

Emanuele Diquattro

Fausto Galmarini

Rossano Giuppa

Aldo Letizia

Paolo Palliola

Enzo Rocca

Fabio Salis

Vignettista: Silvano Gaggero

Proprietà, Redazione e Segreteria:

Associazione Italiana Financial Industry Risk Managers (AIFIRM), Via Sile 18, 20139 Milano

Registrazione del Tribunale di Milano $\mathrm{n}^{\circ} 629$ del 10/9/2004

E-mail: segreteria@ aifirm.it;

Tel. 3896946315

Lunedì - Venerdì h.15-17

Stampa: Algraphy S.n.c. - Passo Ponte Carrega 62-62r, 16141 Genova

Le opinioni espresse negli articoli impegnano unicamente la responsabilità dei rispettivi autori

SPEDIZIONE IN ABBONAMENTO POSTALE AI SOCI AIFIRM RESIDENTI IN ITALIA, IN REGOLA CON L'ISCRIZIONE 


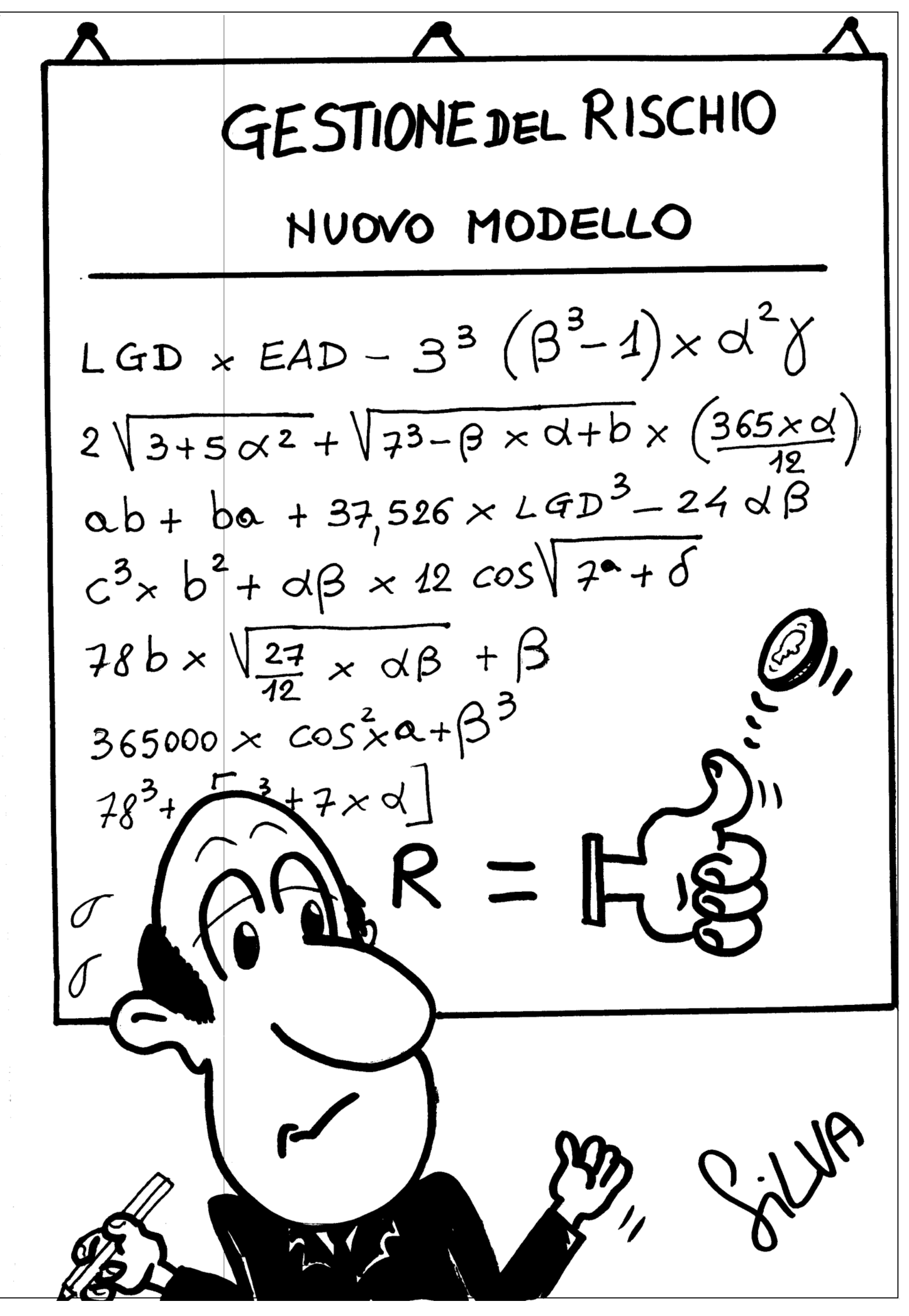




\section{prometeia}

\section{Proficient innovators.}

Proactive thinkers.

\section{Proud souls.}

